J. Electroanal. Chem., 222 (1987) 45-68

Elsevier Sequoia S.A., Lausanne - Printed in The Netherlands

\title{
ANALYSIS OF THE INTERFACIAL ADMITTANCE IN THE CASE OF A TWO-STEP TWO-ELECTRON ELECTRODE REACTION WITH A DIFFUSING INTERMEDIATE, WITH APPLICATION TO THE REDUCTION OF PYRAZINE *
}

\section{RUEDA}

Department of Applied Physical Chemistry, Faculty of Pharmacy, University of Sevilla (Spain)

\author{
M. SLUYTERS-REHBACH and J.H. SLUYTERS
}

Van 't Hoff Laboratory, State University, Padualaan 8, 3584 CH Utrecht (The Netherlands)

(Received 13th June 1986; in revised form 5th November 1986)

\begin{abstract}
A critical evaluation is presented of the analysis of impedance or admittance data in the case of an electrode reaction proceeding by two consecutive one-electron transfers with a stable, solution-soluble intermediate. It is shown that the expression for this case, as derived by Armstrong and Firman, has four limiting forms that have the same frequency dependence as the expression describing a single chargetransfer reaction ("pseudo-Randles behaviour"). Also, the conditions for another simplification, which leads to quarter circles in the complex plane diagram ("Armstrong's equivalent circuit"), are considered. It follows that the special case, where the quarter circle is in the negative quadrant, cannot occur when the system is dc reversible or at equilibrium. For dc irreversible systems, this phenomenon can show up under rather narrow conditions. In addition, it is found that, if these limiting cases are not fulfilled, the general equation often has a frequency dependence that within the practically useful frequency range is hardly distinguishable from that of the single charge-transfer case ("apparent Randles behaviour"). Consequently, the presence of the consecutive mechanism has to be detected from the dc potential dependence of the admittance parameters rather than from the frequency dependence. Finally, it is shown that the general expression corresponds to an equivalent circuit consisting of five elements which, however, have no sensible physical meaning.

These views are applied to the reduction of pyrazine at a DME from $1 M$ aqueous $\mathrm{NaClO}_{4}$ solutions of pH 4.8,5.4 and 6.1. Although only one wave is observed in the dc polarogram, the potential dependence of the transfer resistance and the Warburg coefficient (found as parameters in a case of "apparent Randles behaviour") indicates two consecutive one-electron transfers. The standard rate constants and the transfer coefficients of these two electron transfers are calculated, as well as the rate constant of a homogeneous chemical reaction following the second electron transfer. It is concluded that slow protonation steps largely determine the kinetics of the electron transfers.

The reactants are found to be weakly adsorbed in contrast with the strong adsorption found earlier in strongly acidic solutions.
\end{abstract}

\footnotetext{
‡ Dedicated to the memory of Don Smith.
} 


\section{(I) INTRODUCTION}

The reduction of pyrazine at a mercury electrode appears to be a suitable reaction to show the applicability of the faradaic impedance method in organic electrochemistry. This choice is also based on the biological interest of pyrazine and its derivatives [1,2] and on the fact that its electrochemistry is known in several aspects. Previous studies, using dc and pulse polarography, cyclic and staircase voltammetry, chronoamperometry and electrolysis experiments [3-11] have established that the reduction proceeds in two electron-transfer steps with diffusion of the intermediate under certain circumstances concerning the $\mathrm{pH}$ and solvent composition. The stability of the intermediate is confirmed from electrolysis experiments because of its persistent violet colour [8] and it has been suggested to be the pyridinium radical cation on the basis of ESR experiments [12].

Reversible electrochemical responses to the different perturbation techniques have been observed in strongly acid solutions, but their behaviour becomes more irreversible at higher $\mathrm{pH}$. The homogeneous hydrolysis of the ultimate reduction product, dihydropyrazine, was proposed to explain this $\mathrm{pH}$ dependence, and the rate constant of this reaction was obtained as a function of $\mathrm{pH}$ by chronoamperometry [8].

Knowledge of the kinetics of the heterogeneous process proper is more scarce. In an attempt to obtain quantitative data, Moorhead and Tuite [13] used the dc current data at the foot of the polarographic wave to obtain values of the apparent heterogeneous standard rate constants of the first one-electron reduction step in very acid solutions and of the two-electron process occurring at higher $\mathrm{pH}$. However, the assumptions involved in the analysis are not completely justified because the electrode reactions are rather fast and because of the stability of the intermediate at higher $\mathrm{pH}$. Moreover, although from the $\mathrm{pH}$ dependence of the half-wave potentials it is evident that protons are involved in the reduction processes, their participation in the kinetics was not taken into account.

In a previous study [14] with impedance measurements, at $\mathrm{pH} 0.5$ and 2.8 , we have shown that in these acid media the charge-transfer rates are immeasurably high, while in addition, the reactant, the intermediate and the reduction product are all adsorbed at the mercury/solution interface. The adsorption becomes weaker at higher $\mathrm{pH}$, but is detectable up to $\mathrm{pH} 4$. In the $\mathrm{pH}$ region $3-4$, both finite charge transfer and adsorption occur and consequently the complicated admittance expressions published by Moreira and De Levie [15] and by Birke [16] have to be applied. However, above pH 4, where the adsorption effects are absent or unimportant, the theory of Armstrong and Firman [17] for step-wise reactions with a solution-soluble intermediate should be applicable for the impedance or admittance analysis.

It is the purpose of this paper to perform a quantitative study of the charge-transfer kinetics involved in the reduction of pyrazine in the absence of adsorption and to establish the role played by protons therein. To this end, the reduction has been studied at three $\mathrm{pH}$ values: $4.8,5.4$ and 6.1. At higher $\mathrm{pH}$, the reduction product, dihydropyrazine, becomes too unstable, which will make the frequency dependence 
of the faradaic admittance more complex [18]. Several aspects of the admittance, as regards its frequency and potential dependence, which are of general importance for this type of redox system, will be considered, whereafter these will be compared with our experimental results.

(II) THEORY

(II.1) The frequency and potential dependence of the interfacial admittance in the case of two charge-transfer steps proceeding with finite rates and with a stable intermediate

The expression for the faradaic admittance for the mechanism

$\mathrm{O}+e^{-} \rightleftarrows \mathrm{Y}$

$\mathbf{Y}+e^{-} \rightleftarrows \mathbf{R}$

with diffusing species $Y$ has been derived by Armstrong and Firman [17] and can be written as [19]

$Y_{\mathrm{F}}=\frac{\left[F_{\mathrm{I}}+F_{\mathrm{II}}\right]+\left[2 F_{\mathrm{I}} \lambda_{\mathrm{II}}-2 F_{\mathrm{II}} \lambda_{-\mathrm{I}}+F_{\mathrm{II}} \lambda_{\mathrm{I}}-F_{\mathrm{I}} \lambda_{-\mathrm{II}}\right] s^{-1 / 2}}{1+\left[\lambda_{\mathrm{I}}-\lambda_{-\mathrm{I}}+\lambda_{\mathrm{II}}-\lambda_{-\mathrm{II}}\right] s^{-1 / 2}+\left[\lambda_{\mathrm{I}} \lambda_{\mathrm{II}}-\lambda_{\mathrm{I}} \lambda_{-\mathrm{II}}+\lambda_{-\mathrm{I}} \lambda_{-\mathrm{II}}\right] s^{-1}}$

where $s=\mathrm{i} \omega, \mathrm{i}=\sqrt{-1}$, and $F_{\mathrm{I}}$ and $F_{\mathrm{II}}$ are the partial derivatives $\left(\partial j_{\mathrm{F}, \mathrm{I}} / \partial E\right)_{\mathrm{c}}$ and $\left(\partial j_{F, I I} / \partial E\right)_{c}$ for the two individual steps. On the assumption of linear rate equations:

$j_{\mathrm{F}, \mathrm{I}}=-F\left[k_{\mathrm{I}} c_{\mathrm{O}}-k_{-\mathrm{I}} c_{\mathrm{Y}}\right]$

$j_{\mathrm{F}, \mathrm{II}}=-F\left[k_{\mathrm{II}} c_{\mathrm{Y}}-k_{-\mathrm{II}} c_{\mathrm{R}}\right]$

we have

$$
\begin{aligned}
F_{\mathrm{I}} & =\left(F^{2} / R T\right)\left[\alpha_{\mathrm{I}} k_{\mathrm{I}} \bar{c}_{0}+\left(1-\alpha_{\mathrm{I}}\right) k_{-\mathrm{I}} \bar{c}_{\mathrm{Y}}\right] \\
F_{\mathrm{II}} & =\left(F^{2} / R T\right)\left[\alpha_{\mathrm{II}} k_{\mathrm{II}} \bar{c}_{\mathrm{Y}}+\left(1-\alpha_{\mathrm{II}}\right) k_{-\mathrm{II}} \bar{c}_{\mathrm{R}}\right]
\end{aligned}
$$

where $\bar{c}_{\mathrm{O}}, \bar{c}_{\mathrm{Y}}$ and $\bar{c}_{\mathrm{R}}$ denote the mean surface concentrations and $\alpha_{\mathrm{I}}$ and $\alpha_{\mathrm{II}}$ stand for the respective operational transfer coefficients defined as

$$
\begin{gathered}
\alpha_{\mathrm{I}}=-(R T / F) \mathrm{d} \ln k_{\mathrm{I}} / \mathrm{d} E \\
\alpha_{\mathrm{II}}=-(R T / F) \mathrm{d} \ln k_{\mathrm{II}} / \mathrm{d} E
\end{gathered}
$$

If necessary, the individual forward and backward rate constants can be related by

$$
\begin{aligned}
\ln \left(k_{-\mathrm{I}} / k_{\mathrm{I}}\right) & =(F / R T)\left(E-E_{\mathrm{I}}^{\circ}\right)=\varphi_{\mathrm{I}} \\
\ln \left(k_{-\mathrm{II}} / k_{\mathrm{II}}\right) & =(F / R T)\left(E-E_{\mathrm{II}}^{\circ}\right)=\varphi_{\mathrm{II}}
\end{aligned}
$$

The symbol $\lambda$ in eqn. (1) represents the quantity $-F^{-1} D_{i}^{-1 / 2}\left(\partial j_{\mathrm{F}} / \partial c_{\mathrm{i}}\right)_{c_{1}, E}$ :

$$
\begin{array}{rlrl}
\lambda_{\mathrm{I}} & =D_{\mathrm{O}}^{-1 / 2} k_{\mathrm{I}} & \lambda_{-\mathrm{I}}=D_{\mathrm{Y}}^{-1 / 2} k_{-\mathrm{I}} \\
\lambda_{\mathrm{II}}=D_{\mathrm{Y}}^{-1 / 2} k_{\mathrm{II}} & \lambda_{-\mathrm{II}}=D_{\mathrm{R}}^{-1 / 2} k_{-\mathrm{II}}
\end{array}
$$


With the formulation of the problem by eqns. (1)-(6) it is implicitly assumed that the two charge-transfer steps proceed by relatively simple mechanisms (involving only first-order reactions), and that complications due to, for example, reactant adsorption or coupled homogeneous reactions are absent on the ac time-scale. A possible influence of a coupled homogeneous reaction on the dc time-scale is not excluded, as this will show up in the potential dependence of $\bar{c}_{\mathrm{O}}, \bar{c}_{\mathrm{Y}}$ and $\bar{c}_{\mathrm{R}}$ dictated by the dc process (see the Appendix).

From eqn. (1) it can be seen that the magnitude and the frequency dependence of $Y_{F}$ are essentially controlled by four parameters, which for convenience will be indicated as $R_{\infty}^{-1}, B, C$ and $D$ :

$Y_{\mathrm{F}}=R_{\infty}^{-1} \frac{1+B s^{-1 / 2}}{1+C s^{-1 / 2}+D s^{-1}}=\frac{1}{Z_{\mathrm{F}}}$

$R_{\infty}^{-1}=F_{\mathrm{I}}+F_{\mathrm{II}}$

$B=A_{\mathrm{II}}\left(D_{\mathrm{O}}^{-1 / 2} k_{\mathrm{I}}+2 D_{\mathrm{Y}}^{-1 / 2} k_{-\mathrm{I}}\right)+A_{\mathrm{I}}\left(2 D_{\mathrm{Y}}^{-1 / 2} k_{\mathrm{II}}+D_{\mathrm{R}}^{-1 / 2} k_{-\mathrm{II}}\right)$

$A_{\mathrm{I}}=F_{\mathrm{I}} /\left(F_{\mathrm{I}}+F_{\mathrm{II}}\right) \quad A_{\mathrm{II}}=F_{\mathrm{II}} /\left(F_{\mathrm{I}}+F_{\mathrm{II}}\right)$

$C=D_{\mathrm{O}}^{-1 / 2} k_{\mathrm{I}}+D_{\mathrm{Y}}^{-1 / 2}\left(k_{-\mathrm{I}}+k_{\mathrm{II}}\right)+D_{\mathrm{R}}^{-1 / 2} k_{-\mathrm{II}}$

$D=D_{\mathrm{O}}^{-1 / 2} D_{\mathrm{Y}}^{-1 / 2} k_{\mathrm{I}} k_{\mathrm{II}}+D_{\mathrm{O}}^{-1 / 2} D_{\mathrm{R}}^{-1 / 2} k_{\mathrm{I}} k_{-\mathrm{II}}+D_{\mathrm{Y}}^{-1 / 2} D_{\mathrm{R}}^{-1 / 2} k_{-\mathrm{I}} k_{-\mathrm{II}}$

Equation (7) defines both the faradaic admittance $Y_{\mathrm{F}}$ and the faradaic impedance $Z_{\mathrm{F}}$.

Naturally, the general expressions (8a)-(8e) are to be applied if there is overlap of the two "faradaic regions", i.e. the regions where the individual rate constants of both reactions have significant values. It is useful, however, to distinguish several cases of overlap, and to conclude that in many of them reduced expressions hold. A survey of these cases is visualized in Fig. 1, and the corresponding expressions for $R_{\infty}^{-1}, B, C$ and $D$ are listed in Table 1 , in each case for the intermediate potential region of maximum overlap as well as for the more positive and negative potential regions. It is important to note that the degree of overlap is not only dependent on the difference in the two standard potentials, but also on the relative values of the two individual standard rate constants. For convenience, in Table 1 the diffusion coefficients were taken to be equal: $D_{\mathrm{O}} \approx D_{\mathrm{Y}} \approx D_{\mathrm{R}}=D_{\imath}$.

Generally, it may be expected that the presence of the consecutive mechanism will be revealed by the frequency dependence of $Y_{F}$ or $Z_{F}$ being different from the so-called Randles' behaviour $[19,20]$ characterized by the expression:

$$
Y_{\mathrm{F}}=\frac{1}{R_{\mathrm{ct}}+\sigma(2 s)^{-1 / 2}}=R_{\mathrm{ct}}^{-1} \frac{1}{1+\lambda s^{-1 / 2}}
$$

where $R_{\mathrm{cr}}$ is called the charge-transfer resistance and $\sigma$ is the Warburg asefficient. In the course of the present investigation, we found, surprisingly, that quite often this criterion fails if the data are analysed in the usual frequency range of ca. $70 \mathrm{~Hz}$ to 5 or $10 \mathrm{kHz}$. In other words, pseudo-Randles' or apparent Randles' behaviour is 

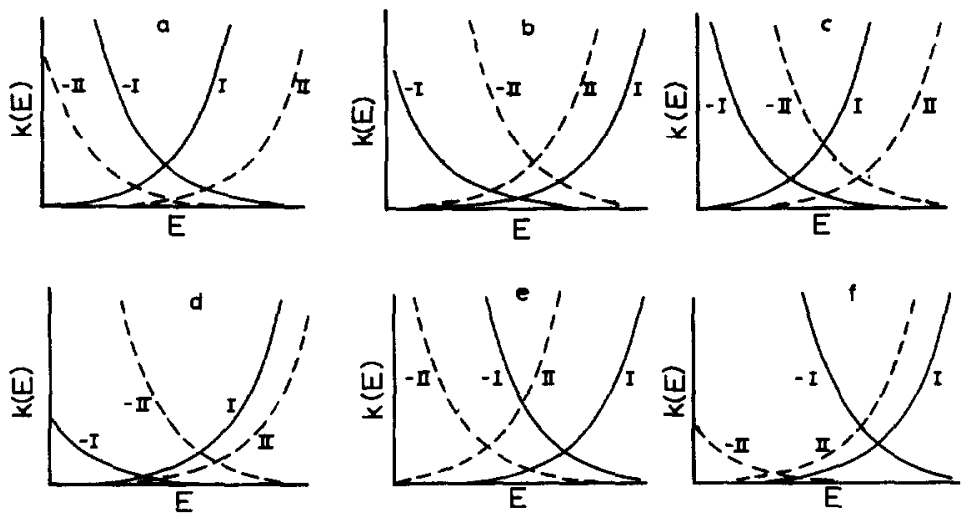

Fig. 1. Schematic representation of the relative magnitude of the rate constants in six cases of overlap. Solid curves: $k_{1}$ and $k_{-1}$; dashed curves: $k_{\mathrm{II}}$ and $k_{-\mathrm{II}}$. See also Table 1.

\section{TABLE 1}

Reduced expressions for the parameters in eqn. (7) corresponding to Fig. 1 at positive, intermediate and negative potentials. $D_{\mathrm{O}} \approx D_{\mathrm{Y}} \approx D_{\mathrm{R}}=D_{\mathrm{i}}$

\begin{tabular}{|c|c|c|c|c|c|}
\hline Conditions & & $R_{\infty}^{-1}$ & $B \times D_{i}^{1 / 2}$ & $C \times D_{1}^{1 / 2}$ & $D \times D_{i}$ \\
\hline $\begin{aligned} \text { (a) } k_{1} & \geqslant k_{1 \mathrm{I}} \\
k_{-\mathrm{I}} & \geqslant k_{- \text {II }}\end{aligned}$ & $\begin{array}{l}\text { pos. } E \\
\text { int. } E\end{array}$ & $\begin{array}{l}\tilde{F}_{1} \\
\tilde{F}_{1}+\tilde{F}_{\mathrm{II}}\end{array}$ & $\begin{array}{l}A_{1} k_{-\mathrm{II}} \approx k_{-\mathrm{II}} \\
A_{1}\left(2 k_{\mathrm{II}}+k_{-\mathrm{II}}\right) \\
+A_{\mathrm{II}}\left(k_{\mathrm{I}}+2 k_{-1}\right)\end{array}$ & $\begin{array}{l}k_{-1}+k_{-\mathrm{II}} \\
k_{\mathrm{I}}+k_{-1}+k_{\mathrm{II}} \\
+k_{-\mathrm{II}}\end{array}$ & $\begin{array}{l}k_{-1} k_{-1 \mathrm{II}} \\
k_{\mathrm{I}} k_{\mathrm{II}}+k_{\mathrm{I}} k_{-\mathrm{II}} \\
+k_{-\mathrm{I}} k_{-\mathrm{II}}\end{array}$ \\
\hline$E_{\mathrm{I}}^{\circ} \gtrless E_{\mathrm{II}}^{\circ}$ & neg. $E$ & $\tilde{F}_{\mathrm{I}}+\tilde{F}_{\mathrm{II}}$ & $A_{11} k_{1}$ & $k_{1}+k_{\mathrm{II}}$ & $k_{1} k_{\mathrm{II}}$ \\
\hline 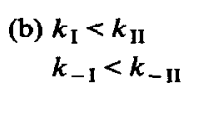 & $\begin{array}{l}\text { pos. } E \\
\text { int. } E\end{array}$ & $\begin{array}{l}\tilde{F}_{1}+\tilde{F}_{1 \mathrm{I}} \\
\tilde{F}_{\mathrm{I}}+\tilde{F}_{1 \mathrm{I}}\end{array}$ & $\begin{array}{l}A_{1} k_{-\mathrm{II}} \\
A_{\mathrm{I}}\left(2 k_{\mathrm{II}}+k_{-\mathrm{II}}\right) \\
+A_{\mathrm{II}}\left(k_{\mathrm{I}}+2 k_{-\mathrm{I}}\right)\end{array}$ & $\begin{array}{l}k_{-\mathrm{II}} \\
k_{\mathrm{II}}+k_{-\mathrm{II}}\end{array}$ & $\begin{array}{l}k_{-1} k_{-1 \mathrm{II}} \\
k_{1} k_{\mathrm{II}}+k_{\mathrm{I}} k_{-\mathrm{II}} \\
+k_{-1} k_{-I I}\end{array}$ \\
\hline$E_{1}^{\circ} \gtrless E_{11}^{\circ}$ & neg. $E$ & $\tilde{F}_{I}+\tilde{F}_{I I}$ & $2 A_{1} k_{11}$ & $k_{11}$ & $k_{\mathrm{I}} k_{\mathrm{II}}$ \\
\hline (c) $\begin{array}{l}k_{\mathrm{I}}>k_{\mathrm{II}} \\
k_{-1}<k_{-\mathrm{II}} \\
E_{\mathrm{I}}^{\mathrm{o}}>E_{\mathrm{II}}^{\circ}\end{array}$ & $\begin{array}{l}\text { pos. } E \\
\text { int. } E \\
\text { neg. } E\end{array}$ & $\begin{array}{l}\tilde{F}_{1} \\
\tilde{F}_{I}+\tilde{F}_{\mathrm{II}} \\
\tilde{F}_{\mathrm{I}}+\tilde{F}_{\mathrm{II}}\end{array}$ & $\begin{array}{l}A_{1} k_{-\mathrm{II}} \approx k_{-\mathrm{II}} \\
A_{1} k_{-\mathrm{II}}+A_{\mathrm{II}} k_{\mathrm{I}} \\
2 A_{\mathrm{I}} k_{\mathrm{II}}+A_{\mathrm{II}} k_{\mathrm{I}}\end{array}$ & $\begin{array}{l}k_{-11}+k_{-1} \\
k_{1}+k_{-11} \\
k_{1}\end{array}$ & $\begin{array}{l}k_{-\mathrm{I}} k_{-\mathrm{II}} \\
k_{\mathrm{I}} k_{-\mathrm{II}} \\
k_{\mathrm{I}} k_{\mathrm{II}}\end{array}$ \\
\hline (d) $k_{\mathrm{I}} \leqslant k_{\mathrm{II}}$ & pos. $E$ & $\tilde{F}_{1}$ & $A_{\mathrm{I}} k_{-\mathrm{II}} \approx k_{-\mathrm{II}}$ & $k_{-1 \mathrm{I}}+k_{\mathrm{I}}+k_{-\mathrm{I}}$ & $k_{-I I}\left(k_{I}+k_{-1}\right)$ \\
\hline$k_{-1} \ll k_{-I I}$ & int. $E$ & $\tilde{F}_{\mathrm{II}}$ & $\begin{array}{l}A_{1}\left(2 k_{1 I}+k_{-\mathrm{II}}\right) \\
+A_{11} k_{\mathrm{I}}\end{array}$ & $k_{\mathrm{I}}+k_{\mathrm{II}}+k_{-\mathrm{II}}$ & $k_{\mathrm{I}}\left(k_{\mathrm{II}}+k_{-\mathrm{II}}\right)$ \\
\hline$E_{\mathrm{I}}^{\circ}>E_{\mathrm{II}}^{\circ}$ & neg. $E$ & $\tilde{F}_{1}+\tilde{F}_{\mathrm{II}}$ & $2 A_{\mathrm{I}} k_{\mathrm{II}}+A_{\mathrm{II}} k_{\mathrm{I}}$ & $k_{\mathrm{I}}+k_{\mathrm{II}}$ & $k_{\mathrm{I}} k_{\mathrm{II}}$ \\
\hline (e) $k_{\mathrm{I}}<k_{\mathrm{II}}$ & pos. $E$ & $\tilde{F}_{1}$ & $\begin{array}{l}A_{1}\left(2 k_{1 I}+k_{-I I}\right) \\
\approx 2 k_{1 I}+k_{-I I}\end{array}$ & $k_{-\mathbf{I}}$ & $k_{-1} k_{-11}$ \\
\hline $\begin{array}{l}k_{-1}>k_{-11} \\
E_{1}^{\circ}<E_{\mathrm{II}}^{\circ}\end{array}$ & $\begin{array}{l}\text { int. } E \\
\text { neg. } E\end{array}$ & $\begin{array}{l}\tilde{F}_{I}+\tilde{F}_{\mathrm{II}} \\
\tilde{F}_{\mathrm{I}}+\tilde{F}_{\mathrm{II}}\end{array}$ & $\begin{array}{l}2 A_{1} k_{1 \mathrm{I}}+A_{1 \mathrm{I}} k_{-\mathrm{I}} \\
2 A_{1} k_{\mathrm{II}}\end{array}$ & $\begin{array}{l}k_{-1}+k_{\mathrm{II}} \\
k_{\mathrm{II}}\end{array}$ & $\begin{array}{l}k_{1} k_{11}+k_{-1} k_{-11} \\
k_{1} k_{\text {II }}\end{array}$ \\
\hline (f) $k_{\mathrm{I}} \leqslant k_{\mathrm{II}}$ & pos. $E$ & $\tilde{F}_{1}$ & $\begin{array}{l}A_{\mathrm{I}}\left(2 k_{1 \mathrm{II}}+k_{-\mathrm{II}}\right) \\
+A_{1 \mathrm{II}} k_{-\mathrm{I}}\end{array}$ & $k_{-1}$ & $k_{-\mathrm{I}} k_{-\mathrm{II}}$ \\
\hline $\begin{array}{l}k_{-\mathrm{I}} \gg k_{-\mathrm{II}} \\
E_{\mathrm{I}}^{\circ}<E_{\mathrm{II}}^{\circ}\end{array}$ & $\begin{array}{l}\text { int. } E \\
\text { neg. } E\end{array}$ & $\begin{array}{l}\tilde{F}_{1} \\
\tilde{F}_{1}+\tilde{F}_{11}\end{array}$ & $\begin{array}{l}2 A_{1} k_{11} \approx 2 k_{11} \\
2 A_{1} k_{1 I}+A_{1 I} k_{I}\end{array}$ & $\begin{array}{l}k_{\mathrm{I}}+k_{-\mathrm{I}}+k_{\mathrm{II}} \\
k_{\mathrm{I}}+k_{\mathrm{II}}\end{array}$ & $\begin{array}{l}k_{\mathrm{I}} k_{1 \mathrm{I}}+k_{-\mathrm{I}} k_{-\mathrm{II}} \\
k_{1 \mathrm{I}} k_{\mathrm{II}}\end{array}$ \\
\hline
\end{tabular}


frequently observed due to the fact that eqn. (7) reduces to the form of eqn. (9) under certain conditions. Then it is necessary to invoke the potential dependence of the obtained $R_{\mathrm{ct}}$ and $a$ values in order to study the kinetics of the consecutive steps. In the following sections, (II.2) to (II.5), we will discuss this matter in more detail.

\section{(II.2) "Real Randles' behaviour"}

Naturally, at the extremes of the faradaic potential region it may occur that overlap is absent or negligible. Then eqn. (7) should reduce to a simpler form, containing only the parameters of one step. Some examples can be found in Table 1 , namely those where it is indicated that $B \approx k_{-11} D_{t}^{-1 / 2}$ : both the numerator and the denominator of eqn. (7) can then be divided by $1+k_{-I I} D_{i}^{-1 / 2} s^{-1 / 2}$, which leads to

$$
Y_{\mathrm{F}}=R_{\infty}^{-1} \frac{1}{1+\left(k_{\mathrm{I}}+k_{-\mathrm{I}}\right) D_{i}^{-1 / 2} s^{-1 / 2}}
$$

i.e. the Randles' equation for the first step.

It should be noted that Table 1 is given to indicate trends, rather than being exhaustive. Therefore there may be more situations in which such a manipulation as that above will be permitted. However, the applicability will always be restricted to small parts of the faradaic potential region.

\section{(II.3) "Pseudo-Randles' behaviour"}

Four limiting cases exist in which eqn. (7) reduces to the form of eqn. (9), but with different meanings of the parameters " $R_{\mathrm{ct}}$ " and " $\sigma$ ". These cases are discussed most conveniently by considering $Z_{\mathrm{F}}$.

Case (i). For high values of the rate constants (ac reversibility) the terms which contain a product $k_{i} k$, dominate the other terms. Thus, if $B s^{-1 / 2} \gg 1$ and $D s^{-1} \gg$ $1+C s^{-1 / 2}$ we obtain from eqn. (7):

$Z_{\mathrm{F}}=R_{\infty}(D / B) s^{-1 / 2}$

and with $s=\mathrm{i} \omega$, the faradaic process is represented by a "Warburg impedance" $\sigma \omega^{-1 / 2}(1-i)$. Naturally, then also the eqns. (3) can be simplified by introducing dc reversible behaviour, i.e. $k_{\mathrm{I}} \bar{c}_{\mathrm{O}}=k_{-\mathrm{I}} \bar{c}_{\mathrm{Y}}$ and $k_{-\mathrm{II}} \bar{c}_{\mathrm{R}}=k_{\mathrm{II}} \bar{c}_{\mathrm{Y}}$. After further elaboration, the rate constants are eliminated from the function $R_{\infty} D / B$ and the Warburg coefficient $\sigma$ can be written as

$\sigma=\frac{R T}{F^{2}\left(2 D_{\mathrm{Y}}\right)^{1 / 2} \bar{c}_{\mathrm{Y}}} \frac{\exp \left(\zeta_{\mathrm{I}}\right)+1+\exp \left(-\zeta_{\mathrm{II}}\right)}{\exp \left(\zeta_{\mathrm{I}}\right)+4 \exp \left(\zeta_{\mathrm{I}}-\zeta_{\mathrm{II}}\right)+\exp \left(-\zeta_{\mathrm{II}}\right)}$

where

$\exp \left(\zeta_{1}\right)=\left(D_{\mathrm{O}}^{1 / 2} / D_{Y}^{1 / 2}\right) \exp \left(\varphi_{I}\right)$ 
$\exp \left(\zeta_{\mathrm{II}}\right)=\left(D_{\mathrm{Y}}^{1 / 2} / D_{\mathrm{R}}^{1 / 2}\right) \exp \left(\varphi_{\mathrm{II}}\right)$

and $\bar{c}_{\mathrm{Y}}$ is given by eqn. (A15) in the Appendix. Equation (12) is identical to the one obtained in ref. 14 for the case involving reactant adsorption. Evidently, the coupling between the diffusion and adsorption does not affect the Warburg coefficient. It is clear that the presence of the two-step mechanism can be deduced only from the potential dependence of $\sigma$, as was shown in ref. 14 .

Case (ii). In fact, since $A_{\mathrm{I}}$ and $A_{\mathrm{II}}$ are dimensionless quantities both $B$ and $C$ are linear functions of the four rate constants. Therefore, it is more realistic to suppose that $D s^{-1}+C s^{-1 / 2} \gg 1$ if $B s^{-1 / 2} \gg 1$. In that case, the faradaic impedance becomes

$Z_{\mathrm{F}}=R_{\infty}\left[(C / B)+(D / B) s^{-1 / 2}\right]$

which is equivalent to a "charge-transfer resistance" $R_{\mathrm{ct}}$ in series with the Warburg impedance of case (i). The potential dependence of $R_{\mathrm{ct}}=R_{\infty} C / B$ and $\sigma=$ $R_{\infty} D / B \sqrt{2}$ can be deduced by combining eqns. (3) and (8) with the expressions for $c_{\mathrm{O}}, c_{\mathrm{Y}}$ and $c_{\mathrm{R}}$ given in the Appendix. The relevant expressions are

$$
\begin{aligned}
& R_{\mathrm{ct}}=\frac{R T}{F^{2}} \frac{D_{\mathrm{Y}}^{1 / 2} k_{\mathrm{II}}^{-1}\left[1+\exp \left(\zeta_{\mathrm{I}}\right)\right]+D_{\mathrm{O}}^{1 / 2} k_{\mathrm{I}}^{-1}\left[1+\exp \left(\zeta_{\mathrm{II}}\right)\right]}{\left[1+2 \exp \left(\zeta_{\mathrm{I}}\right)\right]\left[\alpha_{\mathrm{II}} \bar{c}_{\mathrm{Y}} D_{\mathrm{Y}}^{1 / 2}+\left(1-\alpha_{\mathrm{II}}\right) \bar{c}_{\mathrm{R}} D_{\mathrm{R}}^{1 / 2} \exp \left(\zeta_{\mathrm{II}}\right)\right]+} \\
&+\left[2+\exp \left(\zeta_{\mathrm{II}}\right)\right]\left[\alpha_{\mathrm{I}} \bar{c}_{\mathrm{O}} D_{\mathrm{O}}^{1 / 2}+\left(1-\alpha_{\mathrm{I}}\right) \bar{c}_{\mathrm{Y}} D_{\mathrm{Y}}^{1 / 2}\right] \\
& \sigma=\frac{R T}{F^{2} \sqrt{2}} \frac{1+\exp \left(\zeta_{\mathrm{II}}\right)+\exp \left(\zeta_{\mathrm{I}}+\zeta_{\mathrm{II}}\right)}{\left[1+2 \exp \left(\zeta_{\mathrm{I}}\right)\right]\left[\alpha_{\mathrm{II}} \bar{c}_{\mathrm{Y}} D_{\mathrm{Y}}^{1 / 2}+\left(1-\alpha_{\mathrm{II}}\right) \bar{c}_{\mathrm{R}} D_{\mathrm{R}}^{1 / 2} \exp \left(\zeta_{\mathrm{II}}\right)\right]+} \\
&+\left[2+\exp \left(\zeta_{\mathrm{II}}\right)\right]\left[\alpha_{\mathrm{I}} \bar{c}_{\mathrm{O}} D_{\mathrm{O}}^{1 / 2}+\left(1-\alpha_{\mathrm{I}}\right) \bar{c}_{\mathrm{Y}} D_{\mathrm{Y}}^{1 / 2} \exp \left(\zeta_{\mathrm{I}}\right)\right]
\end{aligned}
$$

with $\bar{c}_{\mathrm{O}}, \bar{c}_{\mathrm{R}}$ and $\bar{c}_{\mathrm{Y}}$ specified by eqns. (A10)-(A12).

Some examples are represented in Figs. 2 and 3 for three values of $E_{\mathrm{II}}^{\circ}-E_{1}^{\circ}$. On the horizontal axes the potentials are given with respect to the standard potential of the overall reaction, $E^{\circ}=1 / 2\left(E_{\mathrm{I}}^{\circ}+E_{\mathrm{II}}^{\circ}\right)$. Because of the coupling between the two steps, the minimum values of $\sigma$ are lower than those for the separate one-electron transfers, but higher than the value for a one-step two-electron transfer. The stability of the intermediate can also be inferred from the width of the $\sigma-E$ and the $R_{\mathrm{ct}}-E$ plots.

Case (iii). For low values of the rate constants the terms including products of the rate constants tend to become negligible, i.e. $D s^{-1}$ and $B s^{-1 / 2}$. Then eqn. (7) reduces to

$Z_{\mathrm{F}}=R_{\infty}\left(1+C s^{-1 / 2}\right)$

again equivalent to Randles' circuit but determined by the "charge-transfer resistance"

$$
\begin{aligned}
R_{\mathrm{ct}}= & \left(R T / F^{2}\right)\left\{k_{\mathrm{I}}\left[\alpha_{\mathrm{I}} \bar{c}_{\mathrm{O}}+\left(1-\alpha_{\mathrm{I}}\right) \bar{c}_{\mathrm{Y}} \exp \left(\varphi_{\mathrm{I}}\right)\right]\right. \\
& \left.+k_{\mathrm{II}}\left[\alpha_{\mathrm{II}} \bar{c}_{\mathrm{Y}}+\left(1-\alpha_{\mathrm{II}}\right) \bar{c}_{\mathrm{R}} \exp \left(\varphi_{\mathrm{II}}\right)\right]\right\}^{-1}
\end{aligned}
$$




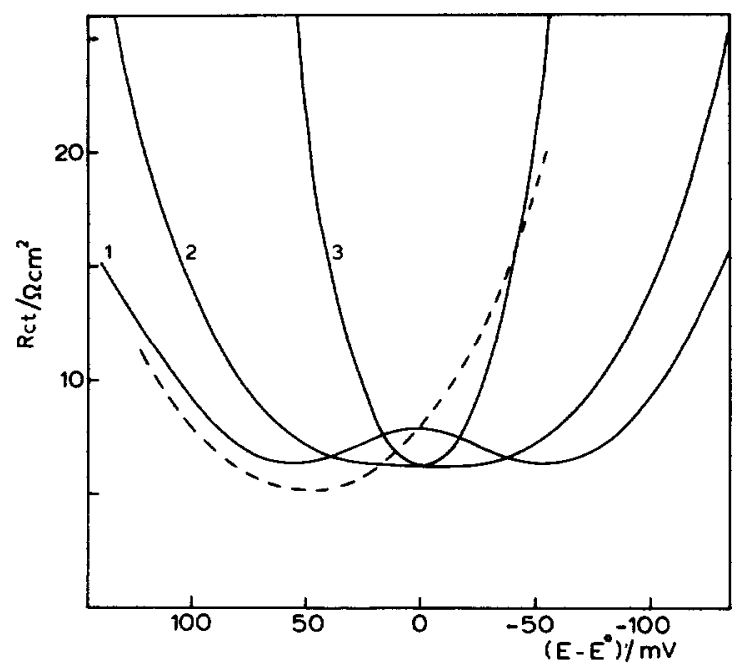

Fig. 2. "Charge-transfer resistance" as a function of the electrode potential. ( $\longrightarrow$ ) Calculated as $R_{\infty} C / B$ with eqn. (14a) for $E_{\mathrm{II}}^{\circ}-E_{\mathrm{I}}^{\circ}$ equal to (1) -100 , (2) -60 and (3) $60 \mathrm{mV}$ with $k_{\mathrm{sI}}=k_{\text {sII }}$ equal to $0.01,0.1$ and 0.5 , respectively. (--) Individual one-electron transfer for $k_{\mathrm{sl}}=0.01$ and $E_{1}^{\circ}-E^{\circ}=+50$ mV.

and the Warburg coefficient

$\sigma=\left(R_{\mathrm{c}} / \sqrt{2}\right)\left\{D_{\mathrm{o}}^{-1 / 2} k_{\mathrm{I}}\left[1+\exp \left(\zeta_{\mathrm{I}}\right)\right]+D_{\mathrm{Y}}^{-1 / 2} k_{\mathrm{II}}\left[1+\exp \left(\zeta_{\mathrm{II}}\right)\right]\right\}$

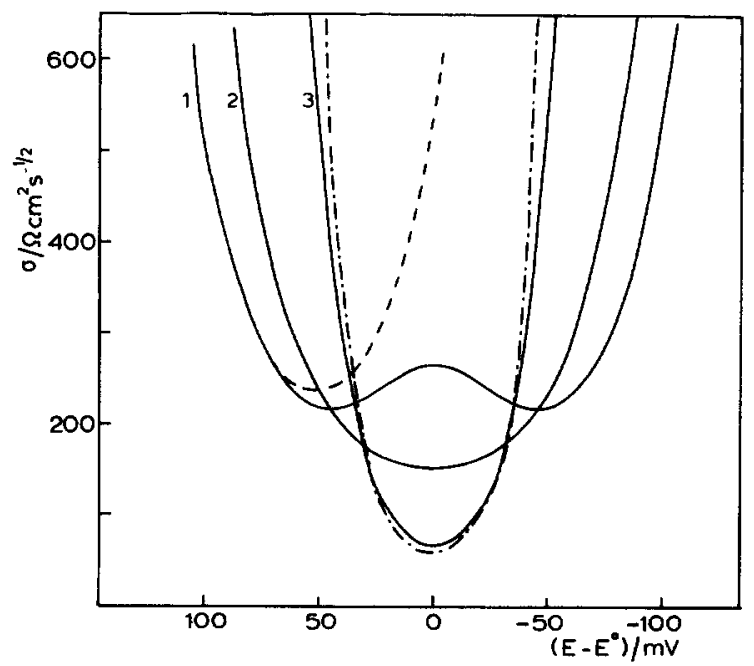

Fig. 3. Warburg coefficient as a function of the electrode potential. (-,---$)$ As Fig. $2 ;(-\cdot-)$ simultaneous two-electron transfer. 
Note that in this case the information on the charge-transfer kinetics is contained not only in $R_{\mathrm{ct}}$ but also in $\sigma$.

Case (iv). A less restrictive situation occurs if the values of the rate constants are such that $D s^{-1} \ll 1+C s^{-1 / 2}$ and $B s^{-1 / 2}<0.1$. Then the expression for $Z_{F}$ can be expanded, which leads to

$Z_{\mathrm{F}}=R_{\infty}\left[1+(C-B) s^{-1 / 2}\right]$

which is still of the Randles type. Note, however, that one can only speak of "Randles behaviour" if $C-B$ is a positive quantity. We will return to this question in the next section, but state here that in the case of dc reversibility $C-B$ is always positive.

It is difficult to formulate quantitative conditions for which one of cases (i)-(iv) holds. Generally, of course, cases (i) and (ii) will be favoured at low frequencies, and cases (iii) and (iv) at high frequencies. In order to estimate the several applicabilities in the total frequency range of $50 \mathrm{~Hz}$ to $10 \mathrm{kHz}$, we performed numerical calculations, comparing the rigorous eqn. (7) with the reduced forms for chosen values of the standard rate constants $k_{\mathrm{s}, \mathrm{I}}$ and $k_{\mathrm{s}, \mathrm{II}}$, defined as the values of $k_{\mathrm{I}}$ at $E_{\mathrm{I}}^{\circ}$ and $k_{\mathrm{II}}$ at $E_{\mathrm{II}}^{\circ}$, respectively. The values of $\alpha_{\mathrm{I}}$ and $\alpha_{\mathrm{II}}$ were both fixed at 0.5 . The conclusions of these calculations are as follows:

Case (i) holds for $k_{\mathrm{s}, \mathrm{I}}$ and $k_{\mathrm{s}, \mathrm{II}}$ larger than unity. Then the rate constants are not
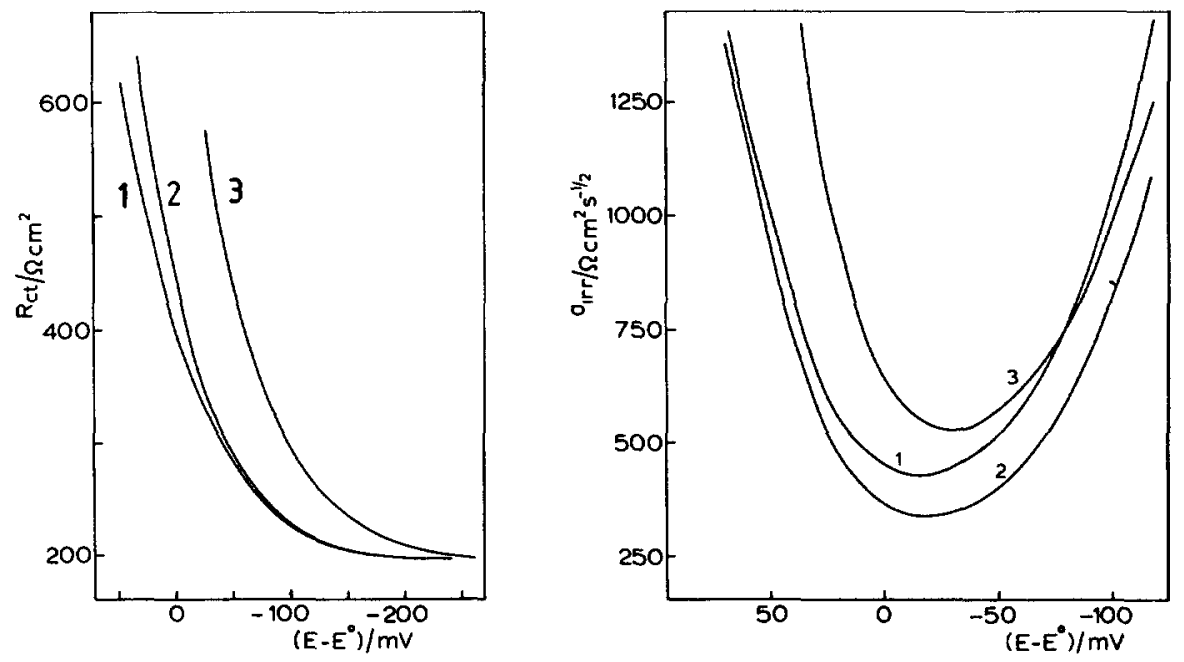

Fig. 4. Charge-transfer resistance as a function of the potential. (- Calculated using the irreversible eqn. (16a) for the same values of the parameters as in Fig. 2.

Fig. 5. Warburg coefficient, as in Fig. 4, calculated using eqn. (16b). 
accessible for the impedance technique, but the stability of the intermediate can be inferred from the shape of the $\sigma-E$ plots.

Case (ii) holds in the entire potential range irrespective of the separation between $E_{\mathrm{I}}^{\circ}$ and $E_{\mathrm{II}}^{\circ}$ if $k_{\mathrm{s}, \mathrm{I}}$ and $k_{\mathrm{s}, \mathrm{II}}$ are larger than $0.2 \mathrm{~cm} \mathrm{~s}^{-1}$. Under this condition, dc reversibility applies, so that $\sigma$ is given by eqn. (12) and $R_{\mathrm{ct}}$ by

$R_{\mathrm{ct}}=\frac{R T}{F^{2}} \frac{k_{\mathrm{I}} D_{\mathrm{Y}}^{1 / 2}\left[1+\exp \left(\zeta_{\mathrm{I}}\right)\right]+k_{\mathrm{II}} D_{\mathrm{O}}^{1 / 2}\left[1+\exp \left(\zeta_{\mathrm{II}}\right)\right]}{k_{\mathrm{I}} k_{\mathrm{II}} \bar{c}_{\mathrm{Y}}\left[\exp \left[\zeta_{\mathrm{I}}+\zeta_{\mathrm{II}}\right)+4 \exp \left(\zeta_{\mathrm{I}}\right)+1\right]}$

The requirements for case (iii) to hold are more restrictive. The condition that $D s^{-1}$ and $B s^{-1 / 2}$ should be negligible whereas $C s^{-1 / 2}$ should not leads to the condition that the standard rate constants are in the range of $10^{-3}-10^{-2} \mathrm{~cm} \mathrm{~s}^{-1}$. Some examples of $R_{\mathrm{ct}}$ and $\sigma$, calculated using eqns. (15) and (16), are given in Figs. 4 and 5. It appears that from these graphs it is more difficult to detect the occurrence of the stable intermediate.

As could be expected, case (iv) is obtained if the rate constants are less than $10^{-3}$ $\mathrm{cm} \mathrm{s}^{-1}$. However, because $\sigma=(C-B) / \sqrt{2}$, eqn. (17) will easily reduce to just $Z_{\mathrm{F}}=R_{\infty}$, and no frequency dependence will develop at all.

\section{(II.4) "Armstrong's equivalent circuit"}

The simplification proposed by Armstrong and Firman [17] essentially relies on the assumption that the influence of mass transport of the reactant $O$ and the final product $R$ is unimportant, in other words, $\lambda_{1} s^{-1 / 2}$ and $\lambda_{-I I} s^{-1 / 2}$ were assumed to be negligible in eqn. (1). Consequently, in eqn. (7), $C$ and $B$ attain a simpler definition and $D$ is eliminated, leading to

$Z_{\mathrm{F}}=R_{\infty} \frac{1+C s^{-1 / 2}}{1+B s^{-1 / 2}}$

In our opinion, this assumption is realistic only if the species $\mathbf{O}$ and $\mathbf{R}$ are present in large and invariable amounts, e.g. in the case of oxidation of a metal to an insoluble oxide. In the case of solution-soluble reactants and products, as studied in the present work, it is clear that all the $\lambda s$ have comparable magnitudes, so that none of them can be neglected selectively.

Nevertheless, it is feasible that for rather low rate constants, $D s^{-1}$ is negligible, leading to eqn. (19) with $C$ and $B$ defined by eqns. (8b), (8c) and (8d). The equivalent circuit designed by Armstrong and Firman [17] applies to this case and so does the complex impedance plane plot for $Z_{F}=Z_{F}^{\prime}-i Z_{F}^{\prime \prime}$. The expressions for $Z_{\mathrm{F}}^{\prime}$ and $Z_{\mathrm{F}}^{\prime \prime}$ are readily obtained by substituting $s=\mathrm{i} \omega$ and separating the real and imaginary parts. It is convenient to write the result in the following notation:

$$
\begin{aligned}
& Z_{\mathrm{F}}^{\prime}=R_{\infty}+R_{\infty} \frac{C-B}{B} \frac{b(b+1)}{b^{2}+2 b+2} \\
& Z_{\mathrm{F}}^{\prime \prime}=R_{\infty} \frac{C-B}{B} \frac{b}{b^{2}+2 b+2}
\end{aligned}
$$


with

$b=(B \sqrt{2}) \omega^{-1 / 2}$

At infinite frequency these expressions reduce to $Z_{\mathrm{F}}^{\prime}=R_{\infty}, Z_{\mathrm{F}}^{\prime \prime}=0$, and at zero frequency, to $Z_{\mathrm{F}}^{\prime}=R_{\infty} C / B, Z_{\mathrm{F}}^{\prime \prime}=0$. The complex plane plot exhibits a "quarter circle", intersecting the horizontal axis at these points, as shown in Fig. 6. Evidently this circle lies in the upper right quadrant if $C / B>1$ and degenerates to a straight line with $45^{\circ}$ slope if $C / B \rightarrow \infty$ (see also case iii in Section II.3). The quarter circle is found in the lower right quadrant if $C / B<1$. This behaviour is the same as that described by Armstrong and Firman [17]. A fourth case was mentioned by these authors, namely a "three-quarter circle" in the upper right and left quadrants, intersecting the horizontal axis at negative $Z_{F}^{\prime}$. It is clear from eqn. (20) that this would require $C / B<0$, which is never realized in our case because in eqn. (8) both $C$ and $B$ are defined as positive quantities.

It is also interesting to examine the conditions to be fulfilled in order that $C / B$ is smaller than unity. To this end, we assume $D_{\mathrm{O}} \approx D_{\mathrm{Y}} \approx D_{\mathrm{R}}$, so that from eqns (8b)-(8d) it follows that

$$
\frac{C}{B}=\frac{k_{1}+k_{-\mathrm{I}}+k_{\mathrm{II}}+k_{-\mathrm{II}}}{A_{\mathrm{II}} k_{\mathrm{I}}+2 A_{\mathrm{II}} k_{-\mathrm{I}}+2\left(1-A_{\mathrm{II}}\right) k_{\mathrm{II}}+\left(1-A_{\mathrm{II}}\right) k_{-\mathrm{II}}}
$$

It is hard to derive from this expression a general condition for which $C / B<1$ is fulfilled. Yet realizing that $A_{\mathrm{II}}$ is a dimensionless quantity between zero and unity, it is easily argued that $C / B$ cannot be less than a certain value. For example,

if $A_{\mathrm{II}} \leq 0.5: \quad C / B \geq k_{\mathrm{II}} / 2 k_{\mathrm{II}}\left(1-A_{\mathrm{II}}\right)=0.5$ to 1

if $A_{11} \geq 0.5: \quad C / B \geq k_{-\mathrm{I}} / 2 k_{-\mathrm{I}} A_{\mathrm{II}}=1$ to 0.5

Clearly, these "absolute minima" are reached only if, with $A_{\mathrm{II}}>0.5, k_{-\mathrm{I}} \gg k_{\mathrm{I}}+k_{\mathrm{II}}$ $+k_{-\mathrm{II}}$, and with $A_{\mathrm{II}}<0.5, k_{\mathrm{II}} \gg k_{1}+k_{-\mathrm{I}}+k_{-\mathrm{II}}$. Such a condition is fulfilled more easily in the case of low rate constants. For example, some values of $C / B$ are represented as a function of the potential in Fig. 7, obtained by numerical calculations choosing favourable values for the standard rate constants.

There are two cases for which $A_{\mathrm{II}}$ attains the special form $A_{\mathrm{II}}=k_{\mathrm{II}} /\left(k_{-\mathrm{I}}+k_{\mathrm{II}}\right)$. This occurs either if $\alpha_{I}=0$ and $\alpha_{I I}=1$, or if the system is dc reversible (then

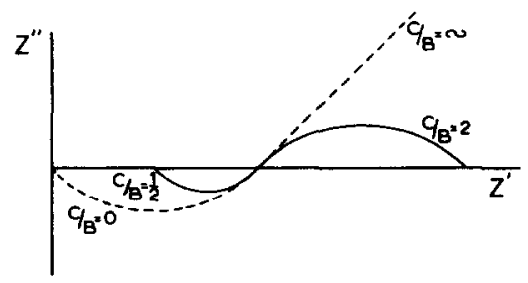

Fig. 6. Complex plane plots of the impedance expressed by eqns. (20a)-(20c). 
$k_{\mathrm{I}} \bar{c}_{\mathrm{O}}=k_{-1} \bar{c}_{\mathrm{Y}}$ and $k_{-\mathrm{II}} \bar{c}_{\mathrm{R}}=k_{\mathrm{II}} \bar{c}_{\mathrm{Y}}$ ) (see eqns. 8c and 3). In these cases, eqn. (21) can be rewritten as

$$
\frac{C}{B}=1+\frac{k_{\mathrm{I}} k_{-\mathrm{I}}+\left(k_{-\mathrm{I}}-k_{\mathrm{II}}\right)^{2}+k_{\mathrm{II}} k_{-\mathrm{II}}}{k_{\mathrm{I}} k_{\mathrm{II}}+4 k_{-\mathrm{I}} k_{\mathrm{II}}+k_{-1} k_{-\mathrm{II}}}
$$

from which it is clear that $C / B>1$ throughout.

It can be concluded that the "negative quarter circle" can be obtained only under $\mathrm{dc}$ polarization of a dc irreversible system, with the additional condition that the forward rate constant of the first step in the mechanism is lower than the forward rate constant of the second step. However, in a limited frequency range a limited part of the circle will be traced in practice. The parameter that controls this part is the parameter $b$ defined in eqn. (20c). For example, the top of the circle is found at the frequency for which $b=\sqrt{2}$, i.e. $\omega=B^{2}$. Consequently, at too low values of the rate constants $B$ is too small to cover a substantial part of the quarter circle. Values of $B$ corresponding to the calculations for Fig. 7 have been plotted in Fig. 8. In addition, in practice the presence of the double-layer capacitance in parallel with the faradaic impedance will obscure the effect drastically.
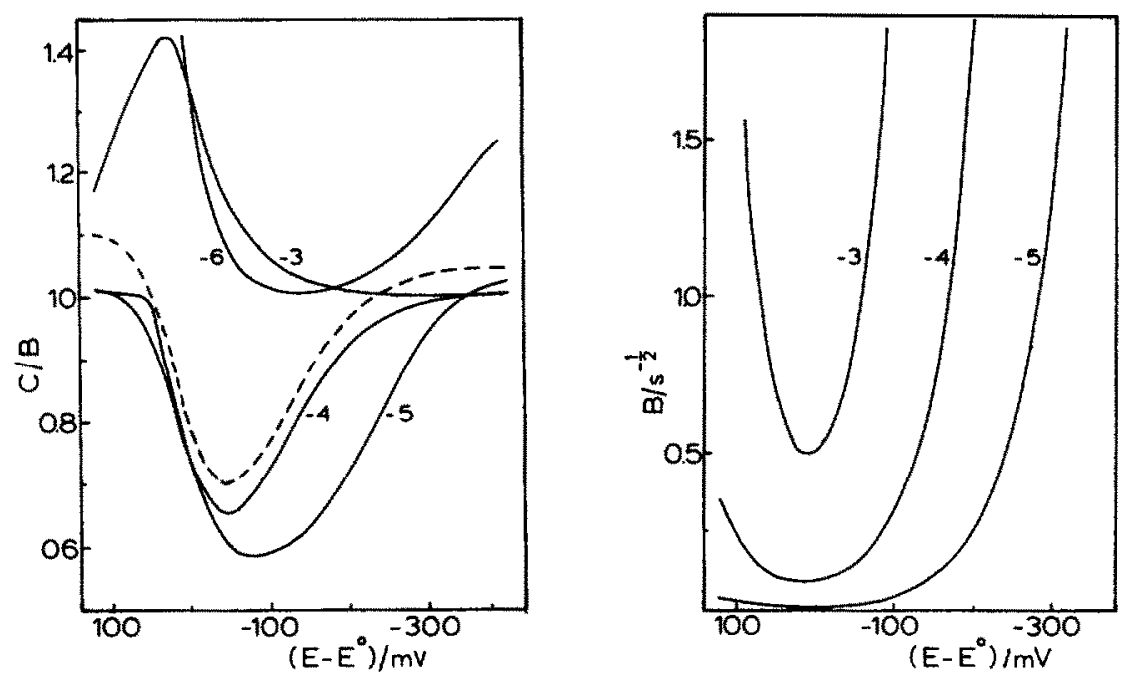

Fig. 7. The function $C / B$ plotted against potential for $E_{\mathrm{I}}^{\circ}=E_{\mathrm{II}}^{\circ}=E^{\circ}, \alpha_{1}=\alpha_{\mathrm{II}}=0.5$. Solid curves: $k_{\mathrm{s}, 1}=10^{-6} \mathrm{~cm} \mathrm{~s}^{-1}, k_{\mathrm{s}, 11}$ varies from $10^{-6}$ to $10^{-3} \mathrm{~cm} \mathrm{~s}^{-1}$ (numbers indicate $\log k_{\mathrm{s}, 11}$ ). Dashed curve: $k_{\mathrm{s}, \mathrm{I}}=10^{-5}, k_{\mathrm{s}, \mathrm{II}}=10^{-4} \mathrm{~cm} \mathrm{~s}^{-1}$.

Fig. 8. The value of $B$ as a function of the potential. Conditions as in Fig. 7. $k_{\mathrm{s}, \mathrm{l}}=10^{-6}, k_{\mathrm{s} . \mathrm{II}}=10^{-5}$, $10^{-4}$ and $10^{-3} \mathrm{~cm} \mathrm{~s}^{-1}$ (numbers indicate $\log k_{\mathrm{s}, \mathrm{H}}$ ). 
(II.5) Frequency analysis with the complete eqn. (7)

An interesting range of (standard) rate constant values, $10^{-3}<k_{\mathrm{s}}<10^{-1} \mathrm{~cm} \mathrm{~s}^{-1}$, is left that is not covered by the cases treated above, so that the complete eqn. (7) is required to perform the analysis. The most convenient expressions are obtained if the complex faradaic impedance is derived by introducing $s=i \omega$, followed by splitting into the real and imaginary components:

$Z_{\mathrm{F}}=R_{\infty}+R_{\infty} \frac{C-B}{B} \frac{b^{2}+b-\mathrm{i} b}{b^{2}+2 b+2}+R_{\infty} \frac{D}{\omega} \frac{b-\mathrm{i}(b+2)}{b^{2}+2 b+2}$

It was not recognized before that this impedance can be described by a relatively simple equivalent circuit, shown in Fig. 9, although this can easily be deduced from eqn. (7). Each of the limiting cases treated above corresponds to a simplification of this circuit by neglecting the influence of one or more of its elements. It should be noted, however, that there are relations between $B, C$ and $D$ through which these quantities are coupled. Therefore, it is not easy to distinguish the time constants of the two parts in series with $R_{\infty}$. In fact, we cannot assign a physical meaning to the elements beyond their resulting from the general eqn. (1).

As it is usual to apply the frequency analysis to admittance data, we have calculated the functions $\omega^{1 / 2} / Y_{\mathrm{F}}^{\prime}$ and $1 / Y_{\mathrm{F}}^{\prime \prime}$, assigning reasonable values to the kinetic parameters. Surprisingly, these functions are found to vary linearly with $\omega^{1 / 2}$ in the frequency range $50-5000 \mathrm{~Hz}$, which can be interpreted as Randles' behaviour at intermediate rate constant $\left(\lambda s^{-1 / 2} \leq 0.2\right)$.

Perusal of the expressions for $Y_{\mathrm{F}}^{\prime}$ and $Y_{\mathrm{F}}^{\prime \prime}$ does not reveal any simple meaning, expressed in analytical form, for the slope and the intercept in these plots. Therefore, this frequency dependence should be designated as an apparent Randles' behaviour. In Fig. 10, some calculated $\omega^{1 / 2} / Y_{\mathrm{F}}^{\prime}$ vs. $\omega^{1 / 2}$ plots are given and compared with the ones calculated for the simplified cases (ii) and (iii). It can be seen that eqn. (13) for the quasi-irreversible case gives higher values for the slope (i.e. higher $\boldsymbol{R}_{\mathrm{ct}}$ values) than the general equation, but quite close $\sigma$ values.

On the contrary, the irreversible case gives somewhat lower $R_{\mathrm{ct}}$ values and more discordant $\sigma$ values. Also, it can be noticed that the applicability of case (ii) is better at higher $k_{\mathrm{s}, \imath}$ values and closer standard potentials of the steps.

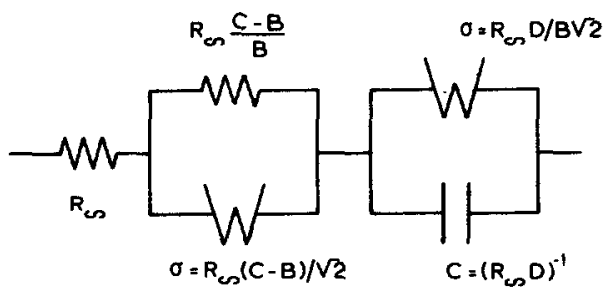

Fig. 9. The equivalent circuit for the step-wise reaction mechanism with a solution-soluble intermediate. 


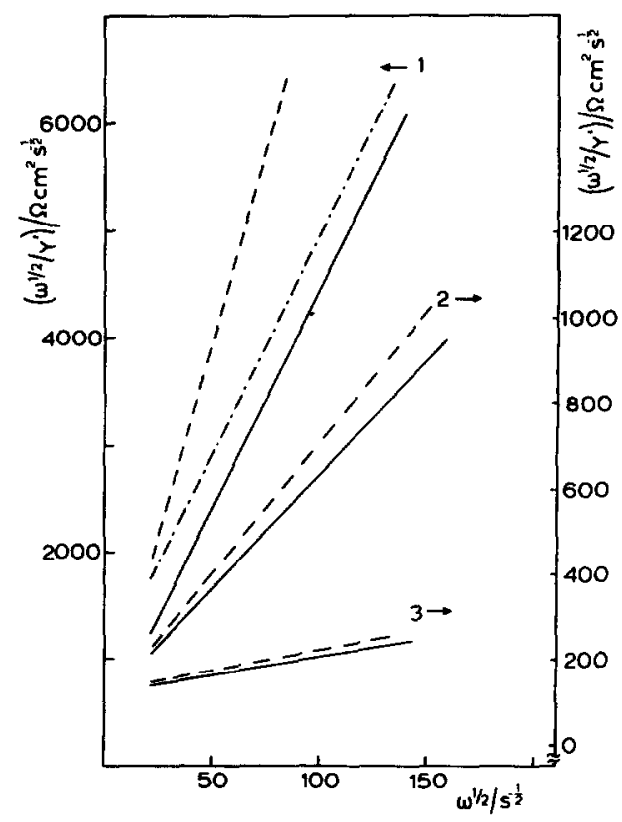

Fig. 10. Calculated plot of $\sqrt{\omega} / Y^{\prime}$ vs. $\sqrt{\omega} .(--)$ Case (ii); $(-\cdot-)$ case (iii); (- $(-)$ general equation. The parameters were chosen as in Fig. 2 with (1) $E-E^{\circ}=35$, (2) $E=E^{\circ}$ and (3) $E=E^{\circ}$.

The conclusion is that the presence of a stable intermediate in a two-electron electrode reaction is not clearly reflected in the frequency dependence of the faradaic admittance. Four different and realistic cases can occur in which this frequency dependence is in accordance with normal Randles' behaviour. Moreover, the $Y_{F}^{\prime} / Y_{F}^{\prime \prime}$ vs. $\omega^{1 / 2}$ plot gives the value unity for the intercept.

In these cases, however, the occurrence of the stable intermediate shows up in the potential dependence of $R_{c t}$ and $\sigma$, which are then different from the theoretical value for a non-diffusing intermediate.

For conditions not covered by cases (i)-(iv), the complete eqn. (1) has to be fitted to the experimental $Y_{\mathrm{F}}(\omega, E)$ values in order to obtain the kinetic parameters of both electron-transfer reactions. However, in the frequency range useful in practice Randles' behaviour apparently occurs. Consequently, also in the intermediate case the information has to be obtained mostly from the potential dependence of $Y_{F}$.

\section{(III) EXPERIMENTAL}

Unless otherwise stated, the experiments were performed with the same cell, electrodes and instrument as in ref. 14.

Supporting electrolyte solutions of $\mathrm{pH} 4.8,5.4$ and 6.1 were prepared from $0.1 \mathrm{M}$ phosphate buffer and $1 \mathrm{M} \mathrm{NaClO}_{4}$. The pyrazine concentration in the cell was 1.05 
$\mathrm{m} M$ at $\mathrm{pH} 4.8$ and $0.90 \mathrm{mM}$ at $\mathrm{pH} 5.4$ and 6.1. Care was taken to avoid evaporation during the experiment.

The cell impedance was obtained at 14 frequencies in the range $80-3000 \mathrm{~Hz}$ and at $10 \mathrm{mV}$ intervals within the faradaic region. The dc current was also recorded at the same potentials. All data were sampled at $4 \mathrm{~s}$ after drop birth.

Similar experiments were carried out in the supporting electrolyte at $\mathrm{pH} 5.4$ in order to obtain the double-layer capacity $C_{\mathrm{d}}$.

(IV) RESULTS AND ANALYSIS

\section{(IV.1) Dc polarograms}

Contrary to what was observed in very acidic solutions, only one wave showed up in these moderate acidic solutions. This wave shifted towards more negative potentials with increasing $\mathrm{pH}$.

Linear $\log \left[j_{\mathrm{F}} /\left(j_{1}-j_{\mathrm{F}}\right)\right]$ vs. $E$ plots were obtained in all cases with a slope of about $35 \mathrm{mV}^{-1}$, which is close to the theoretical reversible value. Therefore no information on the kinetics could be obtained from the dc experiments. The half-wave potential varied with $\mathrm{pH}$, conforming to the requirements for a $3 \mathrm{H}^{+} / 2 e^{-}$reduction. The data obtained from the dc polarograms are reported in Table 2.

\section{TABLE 2}

Characteristics of the dc polarograms

\begin{tabular}{lllll}
\hline $\mathrm{pH}$ & $-E_{1 / 2} / \mathrm{mV}$ & Slope $/ \mathrm{mV}$ & \multicolumn{2}{c}{$10^{5} D_{\mathrm{O}} / \mathrm{cm}^{2} \mathrm{~s}^{-1}$} \\
\cline { 5 - 6 } & & 36 & $\mathrm{a}$ & $\mathrm{b}$ \\
\hline 4.8 & 764 & 34 & 1.6 & 1.2 \\
5.4 & 795 & 37 & 1.6 & 1.2 \\
6.1 & 843 & & 1.5 & 1.1 \\
\hline
\end{tabular}

${ }^{a}$ Diffusion layer model.

b Diffusion layer model with correction for sphericity.

\section{(IV.2) Impedance data}

The real and imaginary admittance components $Y^{\prime}$ and $Y^{\prime \prime}$ were obtained from the cell impedance after subtracting the ohmic resistance $R_{\Omega}$ [19]. The latter was measured at $10 \mathrm{kHz}$ at potentials outside the faradaic region.

Only one peak was observed in the $Y^{\prime}-E$ plots (Fig. 11), with lower $Y^{\prime}$ values for the less acidic solutions indicating that the reaction becomes slower at higher $\mathrm{pH}$.

Unlike the results obtained at $\mathrm{pH} 0.5$ [14], here no reactant adsorption can be inferred from the frequency dependence of the admittance components since the 

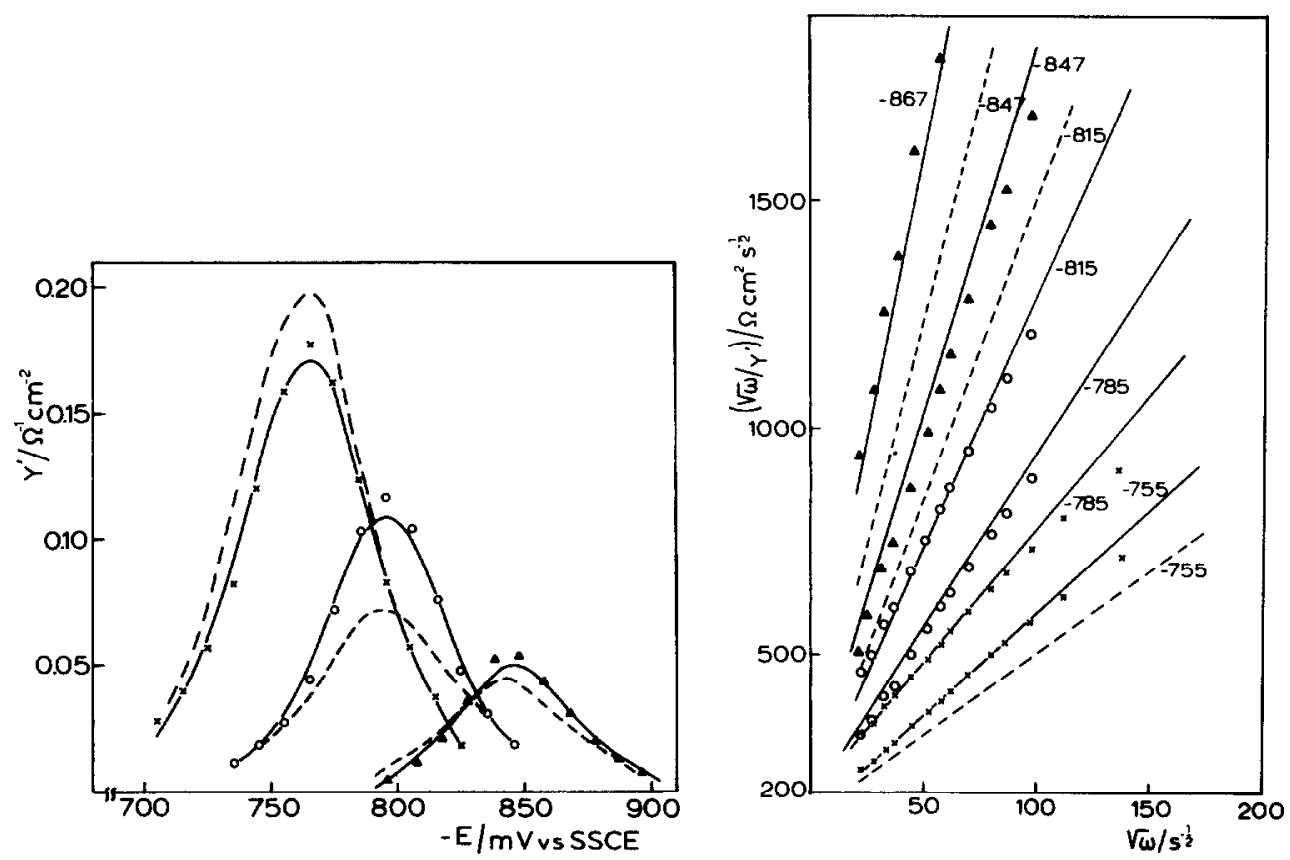

Fig. 11. Experimental value of $Y^{\prime}$ at $1020 \mathrm{~Hz}$ as a function of the electrode potential (vs. SSCE). (X) pH 4.8; (O) $\mathrm{pH} \mathrm{5.4;} \mathrm{( \triangle )} \mathrm{pH} 6.1$. (—) Calculated with the parameters obtained from analysis with general equation; (-- ) the same fitted to case (ii).

Fig. 12. Plot of $\sqrt{\omega} / Y^{\prime}$ vs. $\sqrt{\omega}$ at the electrode potentials indicated. ( $\longrightarrow$ Calculated with the complete equation and the parameters in Table $3 ;(---)$ calculated with the approximate equation for case (ii) and the parameter values in parentheses in Table 3.

$\omega^{1 / 2} / Y^{\prime}$ vs. $\omega^{1 / 2}$ plots are ascending (Fig. 12). These linear plots suggest that Randles' circuit applies. Therefore, the admittance spectra at each potential were analysed according to the equation $[19,20]$

$Y^{\prime}=\frac{\omega^{1 / 2}}{\sigma} \frac{p^{\prime} \omega^{1 / 2}+1}{\left(p^{\prime}\right)^{2} \omega+2 p^{\prime} \omega^{1 / 2}+2}$

where $p^{\prime}=R_{\mathrm{ct}} / \sigma$. Via a computer fit, the values of $R_{\mathrm{ct}}$ and $\sigma$ were obtained at each dc potential.

In Figs. 13 and 14, the resulting $\sigma-E$ and $R_{\mathrm{ct}}-E$ plots are given for the three $\mathrm{pH}$ values. The gradual evolution towards less reversible behaviour at higher $\mathrm{pH}$ clearly shows up from the $R_{\mathrm{ct}}-E$ plots, the $R_{\mathrm{ct}}$ values increasing with $\mathrm{pH}$. Also, the $\sigma_{\min }$ values change in the same way but this $\mathrm{pH}$ dependence cannot be explained on the basis of changes in reversibility.

However, the values of the diffusion coefficient $D_{\mathrm{O}}$, calculated from $\sigma_{\min }$, are $5.0 \times 10^{-6}, 4.7 \times 10^{-6}$ and $4.2 \times 10^{-6} \mathrm{~cm}^{2} \mathrm{~s}^{-1}$ for $\mathrm{pH} 4.8,5.4$ and 6.1 , respectively. 

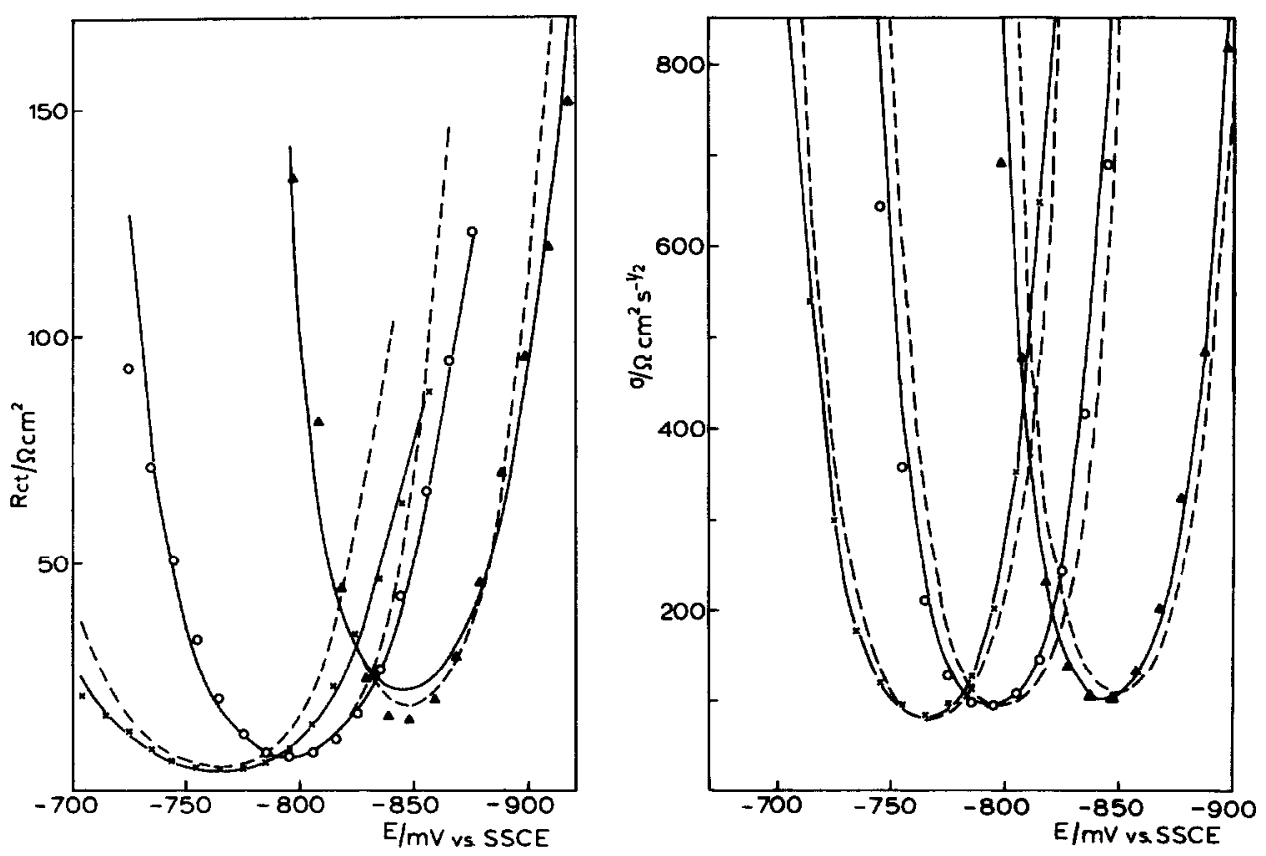

Fig. 13. Charge-transfer resistance as a function of the electrode potential. (- Calculated with the parameters obtained from the optimization of $\sigma$ and $R_{\mathrm{et}}$ to the approximate equation; (- - ) calculated with the parameters obtained from optimization of $Y^{\prime}$ to the general equation, substituted in the approximate solution.

Fig. 14. Warburg coefficient, as in Fig. 13.

These values are much lower than those obtained from the limiting current in the dc polarograms. Somewhat lower $D_{0}$ values have frequently been obtained from ac data, and the difference has been ascribed to the inadequacy of the model used to describe diffusional mass transport to the DME, i.e. the diffusion layer approach with a correction for sphericity. However, here the differences are too large to be explained in this way. Two complications, which were both proved earlier to apply in very acidic solutions [14], can serve to explain the observed behaviour:

(i) the presence of coupled homogeneous reactions; and

(ii) the occurrence of a stepwise mechanism with a stable intermediate.

In Section (II) it has been shown that the latter mechanism leads to more extended $\sigma-E$ and $R_{\mathrm{ct}}-E$ plots and also to higher $\sigma_{\min }$ values (Figs. 2 and 3). Hence the plots in Figs. 13 and 14 can be interpreted on the basis of a mechanism consisting of two one electron transfers with different $\mathrm{pH}$-dependences of their standard potentials such that the intermediate becomes more unstable at higher $\mathrm{pH}$.

Because two protons are transferred in the first step and one in the second, a difference, $\Delta E^{\circ}=E_{\mathrm{II}}^{\circ}-E_{\mathrm{I}}^{\circ}$, of about $70 \mathrm{mV}$ has to be expected at $\mathrm{pH} 4.8$ on the 
basis of the two $E^{\circ}$ values at $\mathrm{pH} 0.5$ in ref. 14. Consequently, although more extended $\sigma-E$ and $R_{\mathrm{ct}}-E$ curves have to be expected, the model cannot account for either the low $D_{0}$ values obtained from $\sigma_{\min }$ or the sequence of $\sigma_{\min }$ values observed with increasing $\mathrm{pH}$, for at higher $\mathrm{pH}$ values the two standard potentials become closer so that $D_{\mathrm{O}}$ should be found to be more equal to the value obtained from the dc limiting current.

Therefore the homogeneous reaction following the second electron transfer found before to exist in strongly acidic solutions [14] can be inferred from the $\sigma-E$ plots in Fig. 13, its rate constant increasing with increasing $\mathrm{pH}$. In this case, the relation between the apparent diffusion coefficient calculated from $\sigma_{\min }$ and its correct value obtained from the limiting current is [21]

$D_{\text {O,app }}^{1 / 2}=4 D_{\mathrm{O}}^{1 / 2} /\left(1+L^{-1 / 2}\right)^{2}$

where $L$ contains the information on the homogeneous reaction as defined in the Appendix. With eqn. (25), the values $L=0.50,0.45$ and 0.35 are obtained at $\mathrm{pH} 4.8$, 5.4 and 6.1 , respectively.

(IV.3) Analysis of the admittance according to the limiting quasi-irreversible case with a stable intermediate (case ii)

Equations (14a) and (14b) (Section II.3) for $R_{\mathrm{ct}}$ and $\sigma$ can be used to calculate the kinetic parameters $k_{\mathrm{sl}}, \alpha_{\mathrm{I}}, k_{\mathrm{sII}}$ and $\alpha_{\mathrm{II}}$ after inserting the expressions for the interfacial concentration $c_{i}$, Appendix eqns. (A10)-(A12).

In order to keep the number of adjustable parameters low, the standard potentials were evaluated from the dc polarogram, assuming the diffusion coefficients to be equal and taking into account that the second electron transfer is followed by the homogeneous reaction [21]

$E_{1 / 2}^{\mathrm{II}}=E_{\mathrm{li}}^{\circ}-(R T / F) \ln L$

Also the separation between $E_{\mathrm{I}}^{\circ}$ and $E_{\mathrm{II}}^{\circ}$ should be in agreement with the separation obtained directly from the two well-resolved waves at very low $\mathrm{pH}$ [14] and the $\mathrm{pH}$ dependences of $E_{\mathrm{I}}^{\circ}$ and $E_{\mathrm{II}}^{\circ}$ to be expected from the participation of two protons and one proton, respectively.

Moreover, the $\sigma$ values at not too negative potentials are hardly affected by slow kinetics, especially at $\mathrm{pH} 4.8$, so that $L, E_{\mathrm{I}}^{\circ}$ and $E_{\mathrm{II}}^{\circ}$ can be varied to get the best fit to the experimental $\sigma-E$ plots around their minimum values. Then the fit of the $R_{\mathrm{ct}}-E$ data to $R_{\infty} C / B$ yields the values of $k_{\mathrm{sI}}, \alpha_{\mathrm{I}}, k_{\mathrm{sII}}$ and $\alpha_{\mathrm{II}}$. As can be seen from Figs. 13 and 14, good agreement between theory and experiment was found in all cases, with the set of parameters given in Table 3 (data in parentheses).

Still, this coincidence should not be considered as final proof for accepting the obtained parameters because the validity of case (ii) used in the analysis is not unquestionable (see Section II). In fact, if these parameters are substituted in the 
TABLE 3

Kinetic parameters and standard potentials, obtained by analysis following the general equation (unbracketed data), compared with the parameters obtained by assuming validity of case (ii) (data in parentheses)

\begin{tabular}{lllllllll}
\hline $\mathrm{pH}$ & $k_{\mathrm{sI}} / \mathrm{cm} \mathrm{s}^{-1}$ & $k_{\mathrm{sI1}} / \mathrm{cm} \mathrm{s}^{-1}$ & $\alpha_{\mathrm{I}}$ & $\alpha_{\mathrm{II}}$ & $-E_{\mathrm{I}}^{\circ} / \mathrm{V}$ & $-E_{\mathrm{iI}}^{\circ} / \mathrm{V}$ & $L$ & $\begin{array}{l}k_{\mathrm{c}} / \\
\mathrm{mm} \mathrm{s}^{-1}\end{array}$ \\
\hline $0.5^{\mathrm{a}}$ & & & & & 0.315 & 0.434 & 0.78 & 0.22 \\
$2.8^{\mathrm{a}}$ & & & & & 0.532 & 0.572 & & \\
4.8 & $0.07-0.13$ & $0.11-0.13$ & $0.05-0.23$ & $0.69-0.90$ & 0.801 & 0.742 & 0.60 & 0.35 \\
& $(0.24)$ & $(0.11)$ & $(0.09)$ & $(0.49)$ & $(0.802)$ & $(0.732)$ & $(0.58)$ & \\
5.4 & $0.05-0.08$ & $0.10-0.16$ & $0.16-0.19$ & $0.70-0.90$ & 0.856 & 0.751 & 0.50 & 0.57 \\
& $(0.12)$ & $(0.04)$ & $(0.34)$ & $(0.72)$ & $(0.849)$ & $(0.749)$ & $(0.45)$ & \\
6.1 & $0.05-0.07$ & $0.07-0.08$ & $0.22-0.26$ & $0.60-0.85$ & 0.936 & 0.776 & 0.35 & 1.4 \\
& $(0.09)$ & $(0.07)$ & $(0.32)$ & $(0.57)$ & $(0.930)$ & $(0.770)$ & $(0.35)$ & \\
\hline
\end{tabular}

a From ref. 14.

general eqn. (1), no satisfactory agreement exists with the experimental values of $Y^{\prime}$ (Fig. 11). A difference also shows up in the $\sqrt{\omega} / Y^{\prime}$ vs. $\sqrt{\omega}$ plots (Fig. 12).

(IV.4) Analysis of the admittance data according to the general eqn. (1)

The complete set of real admittance component values at all potentials and all frequencies was used to obtain the standard rate constants and the transfer coefficients by fitting to the general expression (1) after splitting it into its real and imaginary parts and inserting the expressions (A10)-(A12) for $c_{i}$. The standard potentials and $L$ values were first evaluated as previously explained and then varied slightly in order to get the best fit.

The parameters thus obtained are given in Table 3 (unbracketed numbers). If ranges are reported it should be realized that the left-hand numbers belong together and make up a good fit. The same holds for the right-hand-side numbers.

With these data good agreement with experiment is observed in the $Y^{\prime}$ vs. $E$ (Fig. 11) and the $\sqrt{\omega} / Y^{\prime}$ vs. $\sqrt{\omega}$ plots (Fig. 12).

If the unbracketed data in Table 3 are substituted in the approximate eqns. (14a) and (14b), again the $\sigma$ vs. $E$ and $R_{\mathrm{ct}}$ vs. $E$ plots do not agree with experiment (Figs. 13 and 14), confirming the inadequacy of these equations to describe the electrode reaction.

The imaginary component of the interfacial admittance allows an additional check to be made, for if the model is correct the theoretical $Y_{F}^{\prime \prime}$ values and the experimental $Y^{\prime \prime}$ values are related according to

$Y_{\exp }^{\prime \prime}-Y_{\mathrm{F}}^{\prime \prime}=\omega C_{\mathrm{d}}$

In the absence of adsorption the values of $C_{\mathrm{d}}$ should be equal to the value of the differential double-layer capacity in the supporting electrolyte.

In Fig. 15 the values of $\left(Y_{\text {exp }}^{\prime \prime}-Y_{F}^{\prime \prime}\right) / \omega$ calculated with the parameters in Table 2, which were obtained with the general eqn. (1), are given as a function of the 


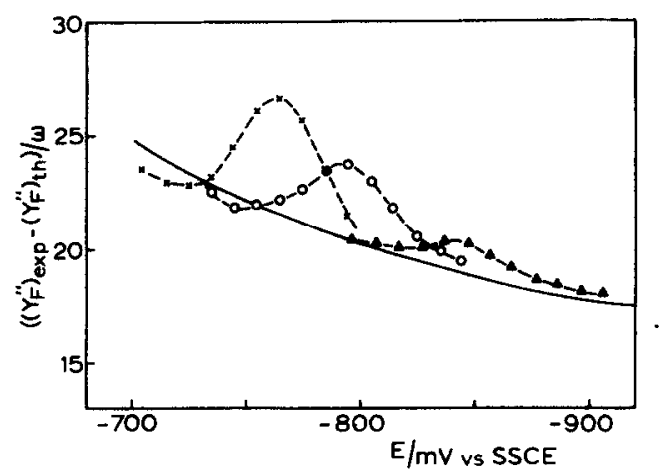

Fig. 15. Test for reactant adsorption as described in the text. (- Experimental double-layer capacity in the supporting electrolyte only.

electrode potential for the three $\mathrm{pH}$ values studied, together with the differential double-layer capacity in the supporting electrolyte. Evidently slight differences show up at potentials close to the half-wave potential, having a close similarity to systems complicated by weak adsorption for which the Randles' circuit applies with an additional parallel capacity [22].

The results in Fig. 15 indicate that adsorption becomes less important at increasing $\mathrm{pH}$ from 4.8 to 6.1 . The strong adsorption at very low $\mathrm{pH}$ [14] fits in this sequence.

From calculations with the equation given by Moreira and de Levie [15] on a one-step finite charge transfer complicated by reactant adsorption it follows that a frequency analysis of $Y^{\prime \prime}$ reveals only the presence of weak adsorption at $k_{\mathrm{s}}>0.1$ $\mathrm{cm} \mathrm{s}^{-1}$ [23]. This suggests that the parameters in Table 3 can be considered unaffected by the adsorption of pyrazine or its reduction products.

\section{(V) DISCUSSION AND CONCLUSIONS}

The results of our analyses, collected in Table 3, show that the standard rate constants of the two electron-transfer steps decrease only slightly with $\mathrm{pH}$. The rate constant $k_{\mathrm{c}}$ of the homogeneous decomposition of dihydropyrazine depends on $\mathrm{pH}$ more clearly, most probably having a minimum at $\mathrm{pH} 2.5$. This behaviour, which is very close to the results of Klatt and Rouseff [8], indicates the occurrence of acid-base catalysed hydrolysis of protonated dihydropyrazine to yield the aminoaldehyde $\mathrm{NH}_{2}-\mathrm{CH}=\mathrm{CH}-\mathrm{NH}-\mathrm{CH}_{2}-\mathrm{CHO}$. With a view to the overall accuracy, the agreement with their chronoamperometric experiments should be considered quantitative. Also, the increase of $k_{\mathrm{c}}$ with $\mathrm{pH}$ explains the apparent irreversibility of the reduction at higher $\mathrm{pH}$ [21].

An interesting feature is the rather extreme value of the transfer coefficient, both of the first and of the second step. The low value of $\alpha_{1}$ suggests the presence of a heterogeneous preceding chemical step largely controlling the overall rate of the first 
reaction. Likewise, the high value of $\alpha_{I I}$ indicates a major contribution of a following chemical step to the kinetics in the second charge-transfer step. These steps could be protonations, which might be involved according to

Process I: $\quad \mathbf{P}_{\mathbf{Y}}+\mathbf{H}^{+} \rightarrow \mathbf{P}_{\mathbf{Y}} \mathbf{H}^{+}$

$$
\begin{aligned}
& \mathbf{P}_{\mathbf{Y}} \mathrm{H}^{+}+e^{-} \rightarrow \mathbf{P}_{\mathbf{Y}} \mathbf{H} \\
& \mathbf{P}_{\mathbf{Y}} \mathrm{H}^{+} \mathbf{H}^{+} \rightarrow \mathbf{P}_{\mathbf{Y}} \mathbf{H}_{2}^{+}
\end{aligned}
$$

Process II: $\quad \mathbf{P}_{\mathbf{Y}} \mathrm{H}_{2}^{+}+e^{-} \rightarrow \mathrm{P}_{\mathbf{Y}} \mathrm{H}_{2}$

$$
\mathrm{P}_{\mathbf{Y}} \mathrm{H}_{2}+\mathrm{H}^{+} \rightarrow \mathrm{P}_{\mathbf{Y}} \mathrm{H}_{3}^{+}
$$

where $\mathrm{P}_{Y} \mathrm{H}_{2}^{+}$is the stable intermediate radical cation [8] and $\mathrm{P}_{\mathrm{Y}} \mathrm{H}_{3}^{+}$is the protonated 1,4-dihydropyrazine.

Expressions for the proton concentration and the potential dependence of the forward rate constants $k_{\mathrm{I}}$ and $k_{\mathrm{II}}$ can be derived following the habitual route described in refs. 24-27 for the two processes (I) and (II), leading to

$\frac{1}{k_{\mathrm{I}}}=\frac{1}{k_{1}\left[\mathrm{H}^{+}\right]}+\frac{K_{\mathrm{I}} \exp \left(\alpha_{2} \varphi_{\mathrm{I}}\right)}{k_{\mathrm{s} 2}\left[\mathrm{H}^{+}\right]}+\frac{K_{\mathrm{I}} \exp \left(\varphi_{\mathrm{I}}\right)}{k_{3}\left[\mathrm{H}^{+}\right]}$

and

$$
\frac{1}{k_{\mathrm{II}}}=\frac{\exp \left(\alpha_{4} \varphi_{\mathrm{II}}\right)}{k_{\mathrm{s} 4}}+\frac{\exp \left(\varphi_{\mathrm{II}}\right)}{k_{\mathrm{s}}\left[\mathrm{H}^{+}\right]}
$$

where $k$ stands for a rate constant, $K$ is the equilibrium constant and $\varphi_{i}=F / R T$ $\left(E-E_{i}^{\circ}\right)$, whereas $\alpha_{2}$ and $\alpha_{4}$ should be close to 0.5 .

A reasoning along these lines implies the adoption of a non-linear potential dependence of $\ln k_{\mathrm{I}}$ and $\ln k_{\mathrm{II}}$ and consequently of a potential dependence of $\alpha_{\mathrm{I}}$ and $\alpha_{\mathrm{II}}$. As a matter of course, these dependences which follow from eqns. (28a) and (28b) can be introduced into the starting eqn. (1) and all the analyses could bc done with it. However, even if we put $\alpha_{2}=\alpha_{4}=0.5$, the two parameters $k_{1}$ and $k_{I I}$ in the original analysis will be replaced by $k_{1}, K_{1}, k_{\mathrm{s} 2}, k_{3}$ and $k_{\mathrm{s} 4}$ and $k_{5}$, respectively.

We feel that with present-day measurement accuracy a fit with this large number of parameters cannot be meaningful; also it should be envisaged that protonation may proceed by protons and by water molecules as parallel processes [27].

\section{APPENDIX}

The mean surface concentrations in the case of two quasi-reversible electron transfers with a stable intermediate followed by a coupled homogeneous reaction

For the mechanism

$$
\begin{aligned}
& \mathrm{O}+e^{-\stackrel{k_{1}}{\underset{k_{-1}}{\rightleftarrows}} \mathrm{Y}} \\
& \mathrm{Y}+e^{-\underset{k_{-1 \mathrm{I}}}{\stackrel{k_{11}}{\rightleftarrows}} \mathrm{R} \underset{k_{-\mathrm{c}}}{\stackrel{k_{\mathrm{c}}}{\rightleftarrows}} \mathrm{Z}}
\end{aligned}
$$


in which the reduced form $R$ is involved in a homogeneous reaction, the expressions for $\bar{c}_{\mathrm{O}}, \bar{c}_{\mathrm{Y}}$ and $\bar{c}_{\mathrm{R}}$ can be derived on the basis of Jacq's treatment [28], starting with $\left[\frac{\partial c_{\mathrm{O}}}{\partial x}\right]_{x=0}=\frac{c_{\mathrm{O}}^{*}-\bar{c}_{\mathrm{O}}}{\delta_{\mathrm{O}}}$

$\left[\frac{\partial c_{\mathbf{Y}}}{\partial x}\right]_{x=0}=-\frac{\bar{c}_{\mathbf{Y}}}{\delta_{\mathbf{Y}}}$

$\left[\frac{\partial\left(c_{\mathrm{R}}+c_{\mathrm{Z}}\right)}{\partial x}\right]_{x=0}=-\frac{\bar{c}_{\mathrm{Z}}-\bar{c}_{\mathrm{R}}}{\delta_{\mathrm{R}}}$

$\left[\frac{\partial\left(K_{\mathrm{c}} c_{\mathrm{Z}}-c_{\mathrm{R}}\right)}{\partial x}\right]_{x=0}=-\frac{K_{\mathrm{c}} \bar{c}_{\mathrm{Z}}-\bar{c}_{\mathrm{R}}}{\delta_{\mathrm{R}}} F(k)$

in which $\delta_{1}$ is the thickness of the diffusion layer for a DME

$\delta_{t}=\left(\frac{3}{7} \pi t D_{t}\right)^{1 / 2}$

$K_{\mathrm{c}}=k_{-\mathrm{c}} / k_{\mathrm{c}} \quad$ and $\quad k=k_{\mathrm{c}}+k_{-\mathrm{c}}$

The rate of the chemical reaction is incorporated in the function

$F\left(k_{\mathrm{c}}\right)=\pi^{1 / 2} \frac{\Gamma\{1+(3 k t / 7)\}}{\Gamma\left\{\frac{1}{2}+(3 k t / 7)\right\}}$

Combination of eqns. (A1) with the flux equations for the mechanism with a stable intermediate:

$j_{\mathrm{F} 1} / F=-D_{\mathrm{O}}\left(\frac{\partial c_{\mathrm{O}}}{\partial x}\right)_{x=0}$

$j_{\mathrm{F} 2} / F=D_{\mathrm{R}}\left(\frac{\partial c_{\mathrm{R}}}{\partial x}\right)_{x=0}$

$\left(j_{\mathrm{F} 2}-j_{\mathrm{F} 1}\right) / F=-D_{\mathrm{Y}}\left(\frac{\partial c_{\mathrm{Y}}}{\partial x}\right)_{x=0}$

leads to the identity

$a_{\mathrm{O}} \bar{c}_{\mathrm{O}}+a_{\mathrm{R}} \bar{c}_{\mathrm{R}}+a_{\mathrm{Y}} \bar{c}_{\mathrm{Y}}=a_{\mathrm{O}} c_{\mathrm{O}}^{*}$

where $a_{0}$ and $a_{\mathrm{R}}$ are defined as

$a_{t}=D_{l}^{1 / 2}(3 / 7 \pi t)^{-1 / 2}$

$a_{\mathrm{R}}=D_{\mathrm{R}}^{1 / 2}(3 / 7 \pi t)^{-1 / 2} L^{-1}$

with

$L=\frac{K_{\mathrm{c}} F(k)+1}{\left(K_{\mathrm{c}}+1\right) F(k)}$ 
The current-voltage equations for the two finite charge transfers

$j_{\mathrm{F} 1}=-F\left(k_{\mathrm{I}} \bar{c}_{\mathrm{O}}-k_{-1} \bar{c}_{\mathrm{Y}}\right)$

$j_{\mathrm{F} 2}=-F\left(k_{\mathrm{II}} \bar{c}_{\mathrm{Y}}-k_{-11} \bar{c}_{\mathrm{R}}\right)$

provide the additional relations needed to obtain the final expressions for the surface concentrations:

$\bar{c}_{\mathrm{O}}=\bar{c}_{\mathrm{Y}}\left[\frac{k_{-\mathrm{I}}}{k_{\mathrm{I}}}+\frac{a_{\mathrm{Y}}}{k_{\mathrm{I}}}+\frac{a_{\mathrm{R}}}{k_{\mathrm{I}}} \frac{a_{\mathrm{R}}}{k_{-\mathrm{II}}+a_{\mathrm{R}}}\right]$

$\bar{c}_{\mathrm{R}}=\bar{c}_{\mathrm{Y}}\left[\frac{k_{\mathrm{II}}}{k_{-\mathrm{II}}+a_{\mathrm{R}}}\right]$

$\bar{c}_{\mathrm{Y}}=c_{\mathrm{O}}^{*}\left[\frac{k_{\mathrm{I}}}{k_{\mathrm{I}}+a_{\mathrm{O}}}\right]\left[\frac{a_{\mathrm{Y}}}{a_{\mathrm{O}}}+\frac{k_{-\mathrm{I}}}{k_{\mathrm{I}}+a_{\mathrm{O}}}+\frac{a_{\mathrm{R}}}{a_{\mathrm{O}}} \frac{k_{\mathrm{II}}}{k_{-\mathrm{II}}+a_{\mathrm{R}}}\right]^{-1}$

The system can be considered as "dc reversible" if the rate constants are much higher than $a_{i}$. Then eqns. (A10)-(A12) reduce to

$$
\begin{aligned}
\bar{c}_{\mathrm{O}} & =\bar{c}_{\mathrm{Y}} \frac{k_{-\mathrm{I}}}{k_{\mathrm{I}}}=\bar{c}_{\mathrm{Y}} \exp \left(\varphi_{\mathrm{I}}\right) \\
\bar{c}_{\mathrm{R}} & =\bar{c}_{\mathrm{Y}} \frac{k_{\mathrm{II}}}{k_{-\mathrm{II}}}=\bar{c}_{\mathrm{Y}} \exp \left(-\varphi_{\mathrm{II}}\right) \\
\bar{c}_{\mathrm{Y}} & =c_{\mathrm{O}}^{*}\left[\frac{a_{\mathrm{Y}}}{a_{\mathrm{O}}}+\frac{k_{-\mathrm{I}}}{k_{\mathrm{I}}}+\frac{a_{\mathrm{R}}}{a_{\mathrm{O}}} \frac{k_{\mathrm{II}}}{k_{-\mathrm{II}}}\right]^{-1} \\
& =c_{\mathrm{O}}^{*}\left[\frac{a_{\mathrm{Y}}}{a_{\mathrm{O}}}+\exp \left(\varphi_{\mathrm{I}}\right)+\frac{a_{\mathrm{R}}}{a_{\mathrm{O}}} \exp \left(-\varphi_{\mathrm{II}}\right)\right]^{-1}
\end{aligned}
$$

\section{ACKNOWLEDGEMENTS}

The authors express their gratitude to Professor M. Sanchez for stimulating their cooperation. This cooperation was supported by the Andalusian Council of Education and Science. The authors are much indebted to Mr. J. Oostveen and Dr. D. Gonzalez Arjona for technical assistance and valuable discussions.

\section{REFERENCES}

1 G. Dryhurst, Electrochemistry of Biological Molecules, Academic Press, New York, 1977.

2 A. Goodman-Gilman, L.S. Goodman and A. Gilman, The Pharmacological Basis of Therapeutics, Macmillan, New York, 1980.

3 L.F. Wiggins and W.S. Wise, J. Chem. Soc.. (1956) 4780.

4 J.M. Hale, J. Electroanal. Chem., 8 (1964) 181.

5 J. Volke, D. Dumanovic and V. Vokova, Collect. Czech. Chem. Commun., 30 (1965) 246.

6 E. Moorhead and D. Britton, Anal, Lett., 1 (1968) 541. 
7 K.B. Wiberg and T.P. Lewis, J. Am. Chem. Soc., 92 (1970) 7154.

8 L.N. Klatt and R.C. Rouseff, J. Am. Chem. Soc., 94 (1972) 7295.

9 J. Volke and S. Beran, Collect. Czech. Chem. Commun., 40 (1975) 2232.

10 M. Maruyama and K. Murakami, J. Electroanal. Chem., 102 (1979) 221.

11 J. Swartz and F.C. Anson, J. Electroanal. Chem., 114 (1980) 117.

12 E.W. Stone and A.H. Maki, J. Chem. Phys., 39 (1963) 1635.

13 E.D. Moorhead and G. Tuite, Anal. Lett., 12 (1979) 421.

14 M. Rueda, M. Sluyters-Rehbach and J.H. Sluyters, J. Electroanal. Chem., 202 (1986) 271.

15 H. Moreira and R. de Levie, J. Electroanal. Chem., 35 (1972) 103.

16 R.D. Birke, J. Electroanal. Chem., 33 (1971) 201.

17 R.D. Armstrong and R.E. Firman, J. Electroanal. Chem., 45 (1973) 3.

18 M. Sluyters-Rehbach and J.H. Sluyters, J. Electroanal. Chem., 23 (1969) 457.

19 M. Sluyters-Rehbach and J.H. Sluyters in J.O'M. Bockris (Ed.), Comprehensive Treatise of Electrochemistry, Vol. 9, Plenum Press, New York, 1984; M. Sluyters-Rehbach and J.H. Sluyters in C.H. Bamford and R.G. Compton (Eds.), Comprehensive Chemical Kinetics, Vol. 26, Elsevier, Amsterdam.

20 M. Sluyters-Rehbach and J.H. Sluyters in A.J. Bard (Ed.), Electroanalytical Chemistry, Vol. 4, Marcel Dekker, New York, 1970.

21 M. Sluyters-Rehbach and J.H. Sluyters, J. Electroanal. Chem., 26 (1970) 237.

22 M. Sluyters-Rehbach and J.H. Sluyters, Rec. Trav. Chim. Pays-Bas, 82 (1963) 553.

23 L. Pospisil, J. Electroanal. Chem., 74 (1976) 369.

24 C.P.M. Bongenaar, A.G. Remunse, M. Sluyters-Rehbach and J.H. Sluyters, J. Electroanal. Chem., 111 (1980) 139.

25 C.P.M. Bongenaar, A.G. Remijnse, E. Temmerman, M. Sluyters-Rehbach and J.H. Sluyters, J. Electroanal. Chem., 111 (1980) 155.

26 C.J. van Velzen, A.G. Remijnse, G.J. Brug, M. Sluyters-Rehbach and J.H. Sluyters, J. Electroanal. Chem., 134 (1982) 87.

27 C.J. van Velzen, A.G. Remijnse, G.J. Brug, M. Sluyters-Rehbach and J.H. Sluyters, J. Electroanal. Chem., 142 (1982) 229.

28 J. Jacq, Electrochim. Acta, 12 (1967) 1. 